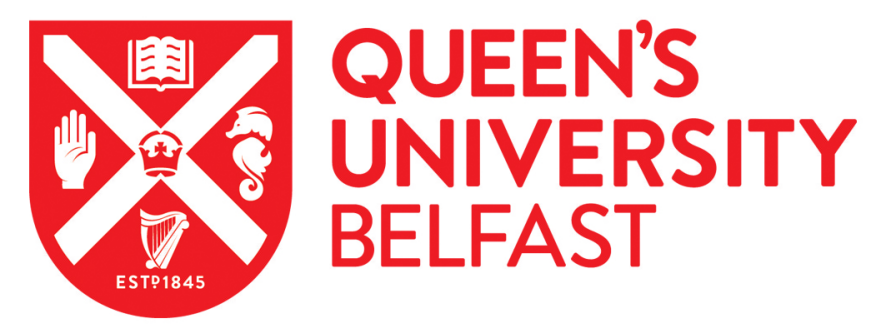

\title{
Electrospun amorphous solid dispersions of poorly water-soluble drugs: A review
}

Yu, D-G., Li, J-J., Williams, G. R., \& Zhao, M. (2018). Electrospun amorphous solid dispersions of poorly watersoluble drugs: A review. Journal of controlled release : official journal of the Controlled Release Society, 292, 91110. https://doi.org/10.1016/j.jconrel.2018.08.016

Published in:

Journal of controlled release : official journal of the Controlled Release Society

Document Version:

Peer reviewed version

Queen's University Belfast - Research Portal:

Link to publication record in Queen's University Belfast Research Portal

Publisher rights

Copyright 2018 Elsevier.

This manuscript is distributed under a Creative Commons Attribution-NonCommercial-NoDerivs License

(https://creativecommons.org/licenses/by-nc-nd/4.0/), which permits distribution and reproduction for non-commercial purposes, provided the author and source are cited.

\section{General rights}

Copyright for the publications made accessible via the Queen's University Belfast Research Portal is retained by the author(s) and / or other copyright owners and it is a condition of accessing these publications that users recognise and abide by the legal requirements associated with these rights.

Take down policy

The Research Portal is Queen's institutional repository that provides access to Queen's research output. Every effort has been made to ensure that content in the Research Portal does not infringe any person's rights, or applicable UK laws. If you discover content in the Research Portal that you believe breaches copyright or violates any law, please contact openaccess@qub.ac.uk. 


\section{Accepted Manuscript}

Electrospun amorphous solid dispersions of poorly water-soluble drugs: A review

Deng-Guang Yu, Jiao-Jiao Li, Gareth R. Williams, Min Zhao

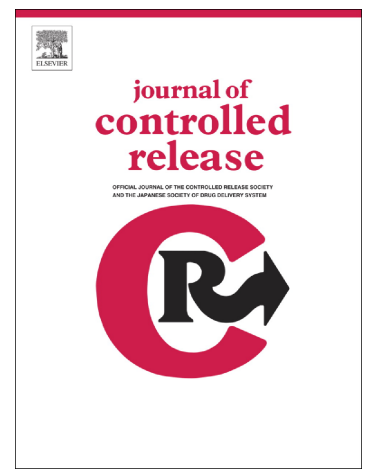

PII:

S0168-3659(18)30482-6

DOI: doi:10.1016/j.jconrel.2018.08.016

Reference: COREL 9425

To appear in: Journal of Controlled Release

Received date: 15 May 2018

Revised date:

7 August 2018

Accepted date:

9 August 2018

Please cite this article as: Deng-Guang Yu, Jiao-Jiao Li, Gareth R. Williams, Min Zhao, Electrospun amorphous solid dispersions of poorly water-soluble drugs: A review. Corel (2018), doi:10.1016/j.jconrel.2018.08.016

This is a PDF file of an unedited manuscript that has been accepted for publication. As a service to our customers we are providing this early version of the manuscript. The manuscript will undergo copyediting, typesetting, and review of the resulting proof before it is published in its final form. Please note that during the production process errors may be discovered which could affect the content, and all legal disclaimers that apply to the journal pertain. 


\section{Electrospun amorphous solid dispersions of poorly water-soluble drugs: A review}

\section{Deng-Guang $\mathrm{Yu}^{1}$, Jiao-Jiao $\mathrm{Li}^{1}$, Gareth R Williams ${ }^{2}$, Min Zhao ${ }^{3, *}$}

1 School of Materials Science \& Engineering, University of Shanghai for Science and Technology, 516 Jungong Road, Shanghai 200093, China.

2 UCL School of Pharmacy, University College London, 29-39 Brunswick Square, London WC1N 1AX, UK.

3 School of Pharmacy, Queen's University Belfast, 97 Lisburn Road, Belfast BT9 7BL UK

* Author for correspondence. Email: m.zhao@qub.ac.uk

\section{Abstract:}

The development of oral dosage forms for poorly water-soluble active pharmaceutical ingredients (APIs) is a persistent challenge. A range of methods has been explored to 
address this issue, and amorphous solid dispersions (ASDs) have received increasing attention. ASDs are typically prepared by starting with a liquid precursor (a solution or melt) and applying energy for solidification. Many techniques can be used, with the emergence of electrospinning as a potent option in recent years. This method uses electrical energy to induce changes from liquid to solid.

Through the direct applications of electrical energy, electrospinning can generate nanofiber-based ASDs from drug-loaded solutions, melts and melt-solutions. The technique can also be combined with other approaches using the application of mechanical, thermal or other energy sources. Electrospinning has numerous advantages over other approaches to produce ASDs. These advantages include extremely rapid drying speeds, ease of implentation, compatibility with a wide range of active ingredients (including those which are thermally labile), and the generation of products with large surface areas and high porosity. Furthermore, this technique exhibits the potential to create so-called 'fifth-generation' ASDs with nanostructured architectures, such as core/shell or Janus systems and their combinations. These advanced systems can improve dissolution behaviour and provide programmable drug release profiles. Additionally, the fiber components and their spatial distributions can be precisely controlled.

Electrospun fiber-based ASDs can maintain an incorporated active ingredient in the amorphous physical form for prolonged periods of time because of their homogeneous drug distribution within the polymer matrix (typically they comprise solid solutions), and ability to inhibit molecular motion. These ASDs can be utilised to generate oral dosage forms for poorly water-soluble drugs, resulting in linear or multiple-phase release of one or more APIs. Electrospun ASDs can also be exploited as templates for manipulating molecular self-assembly, offering a bridge between ASDs and other types of dosage forms.

This review addresses the development, advantages and pharmaceutical applications of electrospinning for producing polymeric ASDs. Material preparation and analysis procedures are considered. The mechanisms through which performance has been improved are also discussed.

Keywords: Poorly water-soluble drug; Amorphous solid dispersion; Electrospinning; Nanofibers; Nano-structure; Core-shell nanofibers; Janus nanostructure; Molecular self-assembly

\section{Introduction}

\subsection{Trends in poorly water-soluble drugs and their solid dispersions}

The development of oral formulations of poorly water-soluble active 
pharmaceutical ingredients (APIs), categorised as class II or IV of the Biopharmaceutical Classification System (BCS), is one of the greatest current challenges in pharmaceutics [1-4]. Continuous efforts have been made to address this issue during the past half century, as evident from simple searches in Web of Science (Figure. 1a); the number of papers matching the search terms of 'insoluble drug' or 'poorly water-soluble drug' is seen to have increased almost exponentially since 1960.

Depending on the properties of the drug(s) of interest, a range of different formulation strategies has been reported over the past decades to improve the solubility and concomitantly the dissolution rate of such molecules. These methods include chemical modification (such as the formation of drug salts and soluble prodrugs) and physical approaches (e.g. micronisation, nanosizing, or polymorph/pseudopolymorph and crystal habit control). Other approaches include tuning of the solvent system used for formulation preparation (e.g. pH adjustment or use of a co-solvent), introducing carrier systems such as liposomes or emulsions, complexation with surfactants or cyclodextrins, and generating solid dispersions (SDs) of the API $[5,6]$. The number of papers reporting the preparation of SDs has rapidly increased over the last decade, as shown in Figure $1 \mathrm{~b}$.

A detailed search of Web of Science for 'amorphous drug solid dispersion' (ASD) yields the trend shown in Figure 1c, demonstrating that the number of publications reporting ASDs has increased rapidly since this concept was first reported by McGinity et al. in 1984 [7]. Figure 1d displays the number of reports of SDs as a proportion of all papers concerning poorly water-soluble drugs, and Figure 1e the ratio of papers reporting ASDs to those reporting SDs. Since 1995, both concepts have gained increasing attention, with ASDs becoming much more prevalent in recent years. These data clearly show that SDs and ASDs have drawn increasing interest in the development of dosage forms for poorly water-soluble APIs. They can additionally be expected to attract further interest in the future as the number of poorly water-soluble drugs developed continuously increases. However, owing to the limited number of pharmaceutical carriers (with acceptable safety profiles) that can be used to ameliorate the solubility issue, new technologies and strategies will be required to ensure that these challenging-to-deliver active ingredients can reach the market.

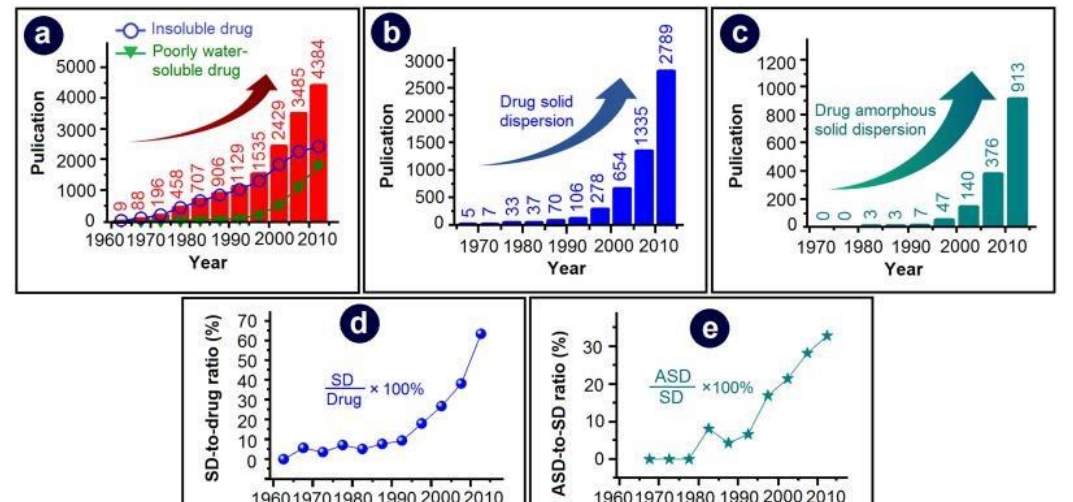


Fig. 1. Data from Web of Science on publications since 1960 concerned with poorly water-soluble APIs and SDs. The results of searches for topics matching (a) 'insoluble drug' and 'poorly water-soluble drug', (b) 'drug solid dispersion' and (c) 'drug amorphous solid dispersion' are shown. The increasing importance of SDs and ASDs can be seen in terms of (d) the ratio of papers matching 'drug solid dispersion' to those matching 'poorly water soluble drug' or 'insoluble drug' and (e) the rise in the proportion of SD papers concerned with ASDs.

\subsection{Generations of SDs}

Similar to general pharmaceutics, the development of SDs relies on having access to both suitable pharmaceutical carriers and technologies for dispersion preparation. The classification of SDs throughout their different development stages is based on the typical pharmaceutical excipients that have been used. First-generation SDs were mainly based on crystalline carriers such as urea, sorbitol and mannitol; in these a crystalline drug is dispersed in the carrier to form a eutectic or monotectic mixture [8,9]. Second-generation SDs mainly comprise binary glass solutions or amorphous solid suspensions, with a poorly water-soluble drug being dispersed in a suitable carrier. These carriers may include:

1) Synthetic polymers such as polyvinylpyrrolidone (PVP), polyvinylpyrrolidone-co-vinyl acetate (PVP-VA), crospovidone (PVP-CL), polyvinyl alcohol (PVA), and polyethylene glycol (PEG);

2) Naturally occurring polymers including hydroxypropylmethyl cellulose (HPMC), hydroxypropylcellulose (HPC), hydroxypropylmethylcellulose phthalate (HPMCP) and hydroxypropylmethyl cellulose acetate succinate (HPMC-AS);

3) Other types of naturally occurring dispersant such as corn starch, potato starch, trehalose, sucrose and inulin [10-13].

Third- generation SDs involve the combined usage of multiple pharmaceutical excipients such as amorphous polymers, surface active agents and/or self-emulsifiers (e.g. poloxamer, Compritol 888 ATO, Soluplus ${ }^{\circledR}$, Gelucire 44/14, sodium lauryl sulphate (SLS), Tween 80 and Inutec SP1) [14-16].

The primary driving force for the transition from first to second-generation SDs 
was the overarching objective of increasing the solubility and dissolution rate of poorly water-soluble APIs. For second-generation SDs, major developments have been achieved in the fabrication technologies used. These have progressed from the initial melting protocols to solvent methods and the combined usage of melting and solvent approaches. Building on these developments, the evolution from second to third-generation SDs aimed to improve further both the dissolution rate and the physical stability of the SDs. The latter is required to prevent precipitation, recrystallisation of the drug within the dispersed system, and other solid phase separations [17].

The simple preparation of an SD or ASD commonly yields a system which dissolves very rapidly. This is helpful for immediate relief of symptoms, but the sustained release of a drug over a prolonged period after oral administration is often desirable and simple SD technology will not delivery such a release profile. Sustained release has the advantage of giving a prolonged therapeutic effect, which reduces the frequency of dosing and improves patient compliance. Sustained-release systems also help in minimising possible side effects, and often allow a reduction in the applied dose while yielding the same therapeutic effect, thereby increasing the effectiveness of an API. As a result of these benefits, fourth-generation SDs of poorly-soluble APIs with controlled release profiles (CR-SD) have drawn increasing attention recently [18]. With a typical CR-SD formulation, the amorphous/molecular dispersion of the API in the carrier improves the drug's dissolution behaviour as in previous generations of SD. However, in contrast to earlier SD iterations, water-insoluble polymers or swellable polymers such as ethyl cellulose (EC), Eudragit ${ }^{\circledR}$ RS or RL, carboxyvinylpolymer (Carbopol $\left.{ }^{\circledR}\right)$ and cross-linked poly(2-hydroxyethyl methacrylate) (PHEMA) are also used in the formulation. These excipients control the drug release, freeing the active ingredients into the dissolution media over an extended time period [19-21].

In recent years, fifth-generation SDs have increasingly been reported. These are characterised by the use of micro- or nanostructures as a means to improve functional performance. The development of these systems has arisen through the adoption of multidisciplinary approaches where SD technology is combined with polymer science, pharmacodynamics, nanoscience and nanotechnology. As discussed by Tran et al. in their review [18], a CR-SD possesses the functions of an SD and also provides controlled release for poorly water-soluble APIs. However, these systems are monolithic homogeneous constructs, which often results in an initial burst release of the drug present at the surface. This can provide an immediately available dose for rapid action, followed by a gradual and continuous release to maintain the plasma concentration of an API over an extended period of time [18]. However, the burst is 
difficult to predict and control, which can compromise the properties of the formulations produced. Fifth-generation SDs aim to overcome this challenge.

The development of novel nanoscale drug delivery systems (DDSs) is dependent on the advancement of nanotechnology and particularly on the fabrication of complex nanostructures [22-27]. To date, the development of nanotechnologies (both 'topdown' and 'bottom-up' methods) has provided a range of useful tools for creating myriad types of complex nanoscale architectures. These include core-shell, Janus, and tri-layer nanostructures generated from both inorganic and organic materials, as well as the polymeric matrices that are frequently used in pharmaceutical applications [2836]. These nanostructures can be explored to create new types of DDS including fifthgeneration SDs. The core-shell structure (with different exterior and inner components) plays an important role in this regard. Both the core and shell compartments can be tailored to have different compositions [37-43]. The main difference between the first four SD generations and the latest fifth generation is that the performance of the former systems typically relies on the physicochemical properties of the carriers/excipients, while the latter provides programmable functional performance both through the carriers used and the production of complex and controllable nanoscale structures.

\subsection{Technologies for fabricating SDs}

During the past half century, there have been a number of excellent review papers on the technologies that have been applied for creating SDs [44-53]. These all tend to involve the solidification of a liquid system. Based on the working fluids, these technologies can be divided into three categories [54-61]:

1) Melting methods (e.g. fusion, hot melt extrusion, and melt agglomeration);

2) Solvent methods (e.g. vacuum drying, spray drying, freeze-drying, cryogenic processing, precipitation using an anti-solvent, co-precipitation, supercritical anti-solvent, and fluid-bed coating technologies);

3) Melt-solvent methods, combining both the aspects stated above.

With all these methods, the process starts from a working fluid and finishes with a solid product with the drug distributed within the carrier. The main differences are in (1) the nature of the energy applied to convert the liquid to the solid state and its efficiency and (2) the structure (morphology and size) of the final products collected. The different types of energy exploited in fabricating SDs and the properties of the final products are summarised in Table 1 . The nature of the energy used and the process efficiency determine the homogeneity of the resultant SDs and the physical state of the drug in the system. These, in combination with the product morphology, 
in turn determine the properties (including stability) and functional performance of the SDs produced and the solid dosage forms generated from them. 
Table 1 A survey of the technologies which can be applied for fabricating SDs of poorly watersoluble APIs. ES = electrospinning/electrospraying.

\begin{tabular}{|c|c|c|c|c|c|c|}
\hline \multicolumn{2}{|c|}{ Applied energy } & \multirow{2}{*}{ Technology } & \multirow{2}{*}{$\begin{array}{l}\text { Feed } \\
\text { format }\end{array}$} & \multicolumn{2}{|c|}{ Products } & \multirow{2}{*}{$\begin{array}{l}\text { Typical } \\
\text { Refs. }\end{array}$} \\
\hline Main & Additive & & & Drug form & Size & \\
\hline \multirow[t]{3}{*}{$\begin{array}{l}\text { Mech- } \\
\text { anical }\end{array}$} & -- & Ball milling & $\begin{array}{l}\text { Powder/ } \\
\text { Liquid }\end{array}$ & $\begin{array}{l}\text { Crystalline/ } \\
\text { Amorphous }\end{array}$ & $\begin{array}{l}\text { Nano/ } \\
\text { Micro }\end{array}$ & {$[3,59]$} \\
\hline & Thermal & Spraying & Solution & $\begin{array}{l}\text { Amorphous/ } \\
\text { Crystalline }\end{array}$ & Micro & {$[53,55]$} \\
\hline & Thermal & $\begin{array}{l}\text { Fluidised bed } \\
\text { coating }\end{array}$ & Solution & $\begin{array}{l}\text { Amorphous/ } \\
\text { Crystalline }\end{array}$ & Micro & {$[52,58]$} \\
\hline \multirow[t]{3}{*}{ Thermal } & Mechanical & Fusion & & $\begin{array}{l}\text { Amorphous/ } \\
\text { Crystalline }\end{array}$ & Macro & {$[8,56]$} \\
\hline & Mechanical & Melt extr & olid & $\begin{array}{l}\text { Amorphous/ } \\
\text { Crystalline }\end{array}$ & Macro & {$[60,61]$} \\
\hline & -- & $\mathrm{F}_{\mathrm{l}}$ & Solution & $\begin{array}{l}\text { Amorphous/ } \\
\text { Crystalline }\end{array}$ & Macro & [75] \\
\hline \multirow[t]{4}{*}{$\begin{array}{c}\text { Electrost } \\
\text { atic }\end{array}$} & -- & lvent ES & Solution & Amorphous & Nano & [63-65] \\
\hline & Therma & Elevated T ES & Solution & Amorphous & Micro & {$[100]$} \\
\hline & Thermal & Melt ES & Melt & Amorphous & Micro & [93] \\
\hline & Thermal & Electrospray & Solution & Amorphous & Micro & [102] \\
\hline \multirow{2}{*}{$\begin{array}{l}\text { Micro- } \\
\text { wave }\end{array}$} & Mechanical & Microwave & Solution & Amorphous & Macro & [57] \\
\hline & $\begin{array}{l}\text { Thermal / } \\
\text { Mechanical }\end{array}$ & Microwave & Melt & Amorphous & Macro & [66] \\
\hline
\end{tabular}


In all of these approaches, the unprocessed melt or solutions consist of components that are dispersed uniformly because of their rapid movement via Brownian motion. Thus, the faster the conversion from fluid to solid state occurs, the better the homogeneity of the product. In a traditional evaporation process, one major concern is that it is often very hard to remove the solvent(s) from the co-precipitates to an acceptable level rapidly and completely, because the co-precipitates become more and more viscous during the 'drying' process. This prevents further evaporation of the residual solvent, resulting in recrystallisation of the drug in the final SDs [62---64]. Other techniques such as electrospinning (where electrical energy is used and there is very rapid evaporation of the solvent) can thus provide superior quality products to those prepared through simple evaporation. Démuth et al. have compared the ASDs prepared using single-fluid electrospinning and the film casting technology with itraconazole (ITR) as a model API and Kollidon ${ }^{\circledR}$ VA64 as the polymeric matrix. Both X-ray diffraction (XRD) and differential scanning calorimetry (DSC) results demonstrated that the electrospun SDs are totally amorphous while the cast products have some crystalline drug content owing to the slow drying process [65].

In order to achieve rapid conversion from liquids to solid products during the preparation of ASDs, two types of energy are often used in combination, with the expectation that the molecular dispersion of the drug in the liquid state can be completely propagated into the solid state to form a solid solution or an amorphous solid suspension. It is this effective combination of different energies that has led to almost all the second, third and fourth-generation ASDs reported. In some methods, three different types of energy have been used in combination for fabricating ASDs. For example, Passerini et al. have prepared praziquantel ASDs through the combined usage of ultrasonic, thermal and mechanical energy [66].

The morphology and size of the SD products are key parameters in their functional performance. According to the Noyes-Whitney equation [67], the dissolution rate $(d X / d t)$ is defined as follows:

$$
d X / d t=(A \times D / \delta) \times(C-X / V)
$$

where $X$ is the amount of drug in solution, $t$ is the time, $A$ is the effective surface area, $D$ is the diffusion coefficient of the drug, $\delta$ is the size of the effective diffusion boundary layer, $C$ is the saturation solubility of the drug and $V$ is the volume of the dissolution medium. It is clear from this equation that the larger the surface area, the faster the dissolution rate. Thus, enlarging the surface area of SD products has contributed to one of the most important routes for enhancing dissolution rate. Even before the surge of nanotechnology in 1990s, the reduction of the particle sizes of drugs (alone or with carriers), was a key approach used by formulation scientists. Techniques such as microcrystal and microparticle technologies give formulations with reduced particle size and consequently larger surface areas [68-71]. The recent 
and rapid development of nanotechnologies has led to increasing numbers of new methods and approaches being introduced for the fabrication of advanced DDSs and ASDs [72-74].

Considering technologies based on freeze- and vacuum drying, the products often have a porous structure and hence a large surface area [75]. Mesoporous silica can be used as an inorganic carrier to prepare ASDs of APIs following the same principles [76-80]. However, the most commonly explored strategies for increasing surface area involve nanosizing drug/carrier systems [81, 82]. Electrospinning, drying and nanofabrication processes all deliver this goal, and all are widely investigated for the preparation of ASDs. From Table 1, it can be seen that when electrostatic energy is applied as the dominant drying force, the products always have a small size, typically at the micro- or nano-scale. When thermal energy is the major drying force, the products are mainly macroscale, which often requires downstream processing methods, such as milling, pelletisation or calendering $[83,84]$.

Exploiting the fact that liquids can readily interact with electric fields [85], electrostatic energy or electrostatic forces have been broadly investigated as useful tools to prepare novel functional materials, particularly those at the nanoscale. Three related technologies, electrospinning, electrospraying and e-jet printing, are usually considered to comprise 'electrohydrodynamic atomisation' (EHDA) processes [8690]. Electrospinning, based on the direct interactions between working fluids and electrical fields, is probably the most popular of these approaches. This technique involves the preparation of a solution of a polymer and a functional component (e.g. an API) in a volatile solvent. The polymer/drug solution is then ejected from a metaltipped syringe towards a collector, and a high potential difference is applied between the two. This can solidify the fluid jets at an extremely fast speed (often at a time scale on the order of $10^{-2} \mathrm{~s}$ ). The products collected commonly comprise nanoscale fibers, which usually assemble randomly into a non-woven fabric with a huge surface area and very high porosity. The properties of these products make electrospinning a perfect candidate for generating ASDs of poorly water-soluble APIs.

In addition to polymer solutions, other liquids such as nanosuspensions, microemulsions and melts can be electrospun into fibers. Thus, electrospinning can provide strategies to build new SD preparation methods across the three categories of melting, solvent and combined melting-solvent methods. Melt electrospinning involves the combined use of thermal energy and electrical energy [91]. In contrast to traditional fusion and hot melt extrusion methods, melt electrospinning can produce fibers with ultrafine diameters, obviating the need for downstream processes, such as pulverising and sieving. The Nagy group carried out a number of investigations into the preparation of ASDs using melt electrospinning and compared these with analogous ASDs fabricated using solution electrospinning and melt blowing, and 
found the products to have broadly similar properties [65, 92, 93]. Melt electrospinning is an organic solvent free process, which may make it particularly attractive for onward applications [92, 93].

A number of opportunities exist to combine electrospinning with other sources of energy as new tools for creating ASDs. Some examples include the combined usage of electrical energy with mechanical forces (force electrospinning) [94, 95] or with thermal and mechanical energy sources (hot air blow electrospinning or electroblowing) [96, 97]. These techniques have been reported but are still only minimally explored in the context of generating ASDs. Another electrospinning process involving the combined use of thermal energy with electrical energy is elevated temperature electrospinning [98, 99]; this heats the working fluid(s) being used to ensure the components remain in solution. Through this approach, electrospun third-generation SDs have been prepared for helicid [100]. Furthermore, other energy sources, such as infrared or sonic energy, can be combined with electrostatic energy to develop new processes for preparing ASDs [101, 102].

\section{Advantages of electrospinning for creating SDs}

\subsection{The electrospinning process}

Electrospinning has been known for more than 100 years but received relatively minimal attention until the 1990s. Since then, the technique has developed very quickly from the simplest experiments using a single fluid to prepare polymer nanofibers or monolithic nanocomposites to double-fluid (coaxial and side-by-side processes) and multiple-fluid spinning (tri-axial, quarternary-axial, multiple side-byside) for producing nanostructures with increasingly complex nanoscale architectures [34, 103-107]. It has also developed from generating small amounts (mg) of material using a single needle to fabrication on the large $(\mathrm{kg})$ scale using needless and highspeed electrospinning [108,109]. Further, the technique has moved from solely being used alone to combined usage with other technologies [110]. Many excellent reviews have considered the key parameters which must be controlled for the implementation of a successful electrospinning process [111-113]. The single-fluid electrospinning process has been very broadly used for the creation of monolithic nanofiber-based ASDs, whereas relatively few works have investigated coaxial electrospinning and multiple-fluid processes for generating structured ASDs.

In this paper, we will not reiterate the findings of other studies [111-113] but will provide a brief consideration of several key issues. Thus, we will briefly discuss the following: 1) the influence of each component in the electrospinning apparatus on the process; 2) the behaviour of working fluids under electrical fields; and, 3) the relationship between electrospinning and electrospraying. 
An electrospinning system provides a platform for electrostatic energy and working fluid(s) to interact with one another. To make full use of the electrostatic energy, two electrodes are required: one connected to a high voltage power supply applying a potential difference to the working fluids, and another (usually grounded) linked to the fiber collector. A syringe pump or compressed air driver is generally used to deliver the working fluids. A flat-tipped metal needle (spinneret) is typically used to guide the fluid as it is ejected. Among these components, the spinneret is the most important, not only because it guides the working fluids to form a Taylor cone and initiate the electrospinning process, but also because it provides a template for the creation of different types of nanostructure from multiple working fluids [114,115]. The literature contains many reports of spinneret and collector design (particularly for fiber alignment) [116,117]; however, relatively few studies have investigated the high-voltage power supply and fluid-driving systems, perhaps reflecting the fact that the latter are generally standard commercial components. Balogh et al. compared alternating current and direct current power supplies for the preparation of electrospun ASDs from solutions of carvedilol and polymer excipients, such as Eudragit E, Eudragit L100-55 and PVP K90. The fibers prepared using the two different power supplies have almost identical properties and functional performance, but the use of an alternating current power supply can significantly increase the productivity of the electrospinning process [118].

In the implementation of electrospinning, the key prerequisite is to effectively channel the electrostatic energy to the working fluids. This does not necessarily mean that the spinneret must consist of a conductive material $[119,120]$. However, in the majority of publications the spinnerets are prepared from stainless steel capillaries, despite the fact that a metal spinneret can often exert a negative influence on the behaviour of the working fluids [121,122]. Some recent publications reveal that spinnerets coated with anti-static polymers such as Teflon [123] or polyvinyl chloride have several advantages [124]: 1) their anti-static properties allow them to minimise any loss of electrical energy from the working fluids to the environment, and thus the electrical field is exploited effectively; 2) the reduced interfacial interactions between the spinneret and the working fluid makes it easier for a charged fluid jet to form; 3 ) the non-stick nature of the polymers results in smaller attractive forces between the working fluids and spinneret, preventing the possible formation of semi-solid materials on the spinneret and clogging. Thus, polymer-coated spinnerets can create fibers with narrow and uniform size distribution [125].

Although electrospinning is a simple and straightforward process, it involves a complex interplay between fluid dynamics, electrodynamics and rheology. Electrospinning comprises four successive steps: formation of a Taylor core at the nozzle of the spinneret; the ejection of a polymer jet from the cone; the bending and 
whipping of the polymer jet because of its unstable regions; and, the collection of solid nanofibers (Figure 2). Spinning commences when the electrical force applied to the working solution overcomes the surface tension, resulting in the ejection of a thin liquid jet. The semi-vertical angle of the Taylor cone $(\theta)$ (i.e. the sharpness of the hyperboloid) depends on the elastic forces and surface tension of the fluid and often lies in the range of $32^{\circ}<\theta<46^{\circ}$ [126]. However, in some coaxial electrospinning processes (these use a concentric spinneret, with one fluid nested inside another) where the outer fluid was a pure solvent, the straight jet was emitted at a semi-vertical angle of $57^{\circ}$ from the Taylor cone. This is thought to arise since the process is easily initiated because of reduced surface tension and viscoelastic force [127]. The capillary forces and interactions between the working fluids and the inner wall of the spinneret also influence the formation of the Taylor cone [122-125].

The bulk of the charged fluid undergoes solvent evaporation in the unstable regions, where it is subjected to a series of electric forces (Figure 2). The attractive force between the two electrodes $(E)$ drives the fluid from the spinneret to the collector, but the repulsive forces between two adjacent bending coils $(F \mathrm{c})$ counteract this and slow down the process. A third force, the Coulomb repulsion force $(\mathrm{Fe})$, acts to accelerate the drawing of the fluid jet. With the jet gradually thinning, its continuously increasing surface area will accelerate solvent evaporation and fluid jet solidification to generate nanofibers. Thus, the additives in polymer solutions used for spinning, such as drug molecules, salts, and surfactants, exert a certain influence on the process [128-133], and on the properties of the resultant fibers. This has not been widely explored, and only a few publications describe the influence of APIs on the electrospinning of polymer solutions [111,112].

Electrospraying is a similar process to electrospinning, except that in place of a polymer jet charged droplets are ejected from the spinneret, resulting in the formation of spherical particles. Electrospinning shares characteristics of both electrospraying and the conventional solution dry spinning of fibers [134], and no clear boundary exists between electrospinning and electrospraying. When the viscosity of the working fluid used for electrospinning is low, due to lack of physical entanglements of polymer chains, the products typically adopt a beads-on-a-string morphology, which combines characteristics of both electrospun and electrosprayed materials $[135,136]$. Beads-on-a-string properties may be useful for drug delivery and controlled release because of such structures may be regarded as hybrids of nanofibers and micro-particles; however, researchers usually seek to avoid this phenomenon.

The working fluids for electrospraying have lower viscoelasticity than those used for spinning. As shown in Figure 2, electrospraying first creates near-monodisperse droplets, which rapidly shrink as a result of the evaporation of solvents caused by the 
Coulombic forces $(\mathrm{Fe})$. The large surface areas of the micro-droplets allow complete solvent removal in favourable instances. If the solvent in the droplets does not evaporate effectively, thin films will form on the collector [137,138]. The entire process is very fast and is similar in speed to electrospinning. Electrospraying can also be used to produce ASDs, in the form of micro or nanoparticles. For instance, $\mathrm{Yu}$ et al. reported the combined use of electrospraying and electrospinning for creating biphasic controlled release nanofibers, which are essentially fifth-generation ASDs [127]. Both electrospinning and spraying are at an early stage of development, and their numerous applications - either alone or in tandem with other processes - can be envisaged to create a wide range of complicated nanostructures and ASDs for APIs with low solubility in water.

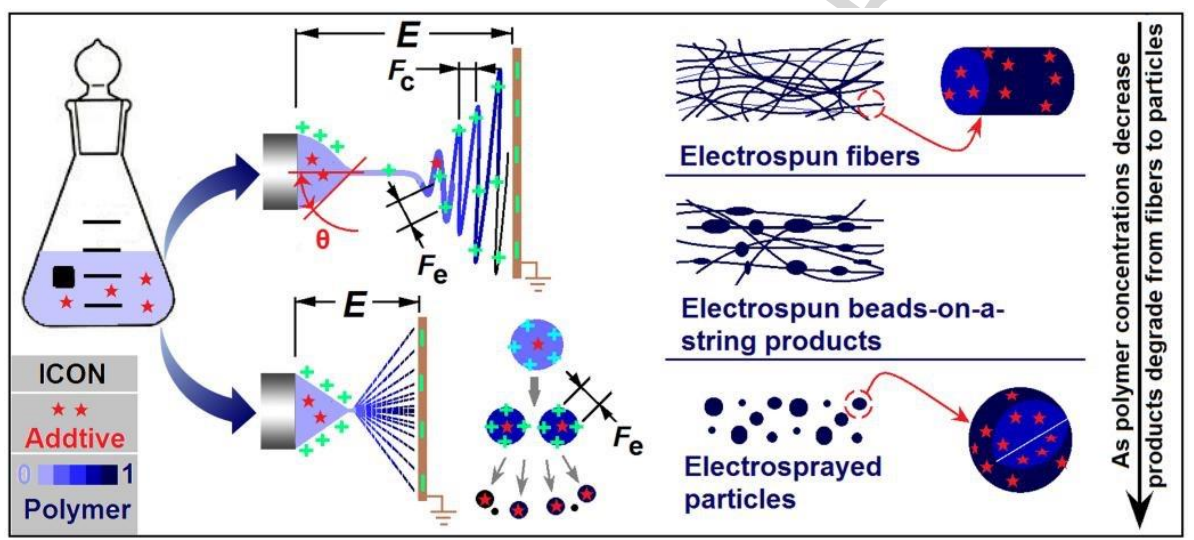

Fig. 2. A schematic of single-fluid electrospinning and electrospraying and their use for preparing monolithic composites at the micro-/nano-scale.

\subsection{Conceiving a SD for a poorly water-soluble drug}

Electrospinning has been widely explored in a number of scientific fields [139]. The potential applications of this method were first reported by Reneker in 1995 [140], and a Web of Science search for 'electrospinning' as the topic yields 19,272 publications within the last five years (see Figure 3) - a daily average of more than ten publications. Electrospun fiber-based ASDs have still not been rigorously investigated, however, especially with regard to their onward commercialisation. There are three interrelated issues that need careful consideration in such applications: 1) the preparation of suitable working fluids with high electrospinnability; 2) the optimisation of the key processing parameters; 3 ) the conversion of fiber mats into an appropriate solid dosage form (Figure 3).

In designing electrospun SDs of poorly water-soluble APIs, the first step is to determine the physicochemical properties of the API, particularly its solubility in a series of commonly encountered organic solvents such as ethanol, methanol, 
chloroform, acetone and acidic/basic aqueous solutions. A strong understanding of physicochemical properties ensures that the selected polymer carriers are compatible and miscible with the API, and allows the user to devise a way to enhance the solubility and dissolution rates and provide a controlled release profile. For the fabrication of API-loaded fibers, the basic principles for the selection of carriers are as follows:

1) Hydrophilic polymers can be used for providing fast dissolution of poorly water-soluble drugs;

2) Insoluble polymers can be exploited to provide sustained release profiles;

3) Certain slowly bio-degradable polymers can be applied to give long-term release in tissue engineering or implant DDSs.

For the preparation of electrospun ASDs of poorly water-soluble drugs, the most commonly explored polymers are water-soluble materials such as PVP, PEO, PVA and HPMC [141-143]. However, water-insoluble polymers have also been exploited in developing new types of ASDs which release the drug in a controlled manner to give improved bioavailability [144]. These insoluble polymers are expected to play an important role in the development of advanced electrospun ASDs. In doing this, we can build on the experiences of researchers working on more traditional formulation types. Computational approaches can also be a useful tool for selecting the appropriate polymeric carrier for APIs [145,146].

In addition to the API and polymer carrier, the solvent system should be carefully considered in preparing working fluids. A single-fluid electrospinning process can prepare second-generation ASDs only if the solvent dissolves both the drug and polymer carrier, and the resultant solution has good spinnability [147]. Otherwise, the drug and polymer must form a spinnable melt and then be converted into an ASD through melt electrospinning [148]. Building on simple binary ASDs, the addition of other pharmaceutical excipients (such as surfactants and sweeteners) to the working fluids can create third-generation ASDs. For example, third-generation ASDs of ferulic acid in PVP nanofibers have been prepared containing sodium dodecyl sulfate and sucralose as surfactant and sweetener additives, respectively. The system not only yielded an improved dissolution rate but also enhanced porcine mucosa permeation performance [149].

Identifying a solvent that can meet all the requirements detailed above is not always easy, and may be impossible. In these cases, one may wish to try different strategies, including a mixed solvent system, and/or altering the electrospinning process. There are many reports of the former. For example, the poorly water-soluble drug quercetin is insoluble in most simple organic solvents but has good solubility in N,Ndimethylacetamide (DMAc). However, ethyl cellulose, often a desired polymer 
carrier, is not spinnable as a DMAc solution. Thus, a mixture of DMAc and ethanol was used for electrospinning to fabricate a controlled-release SD [123]. N.N'Dimethyiformamide (DMF, boiling point $153{ }^{\circ} \mathrm{C}$ ), DMAc $\left(166{ }^{\circ} \mathrm{C}\right)$ and dimethyisulfoxide (DMSO, $189{ }^{\circ} \mathrm{C}$ ) are common aprotic solvents that can dissolve most hydrophobic APIs. However, the high boiling point and low vapour pressures of these solvents make them difficult to process using electrospinning. Thus, typically only small amounts of these solvents are mixed with volatile solvents, such as acetone and ethanol, for electrospinning.

In terms of the electrospinning process itself, the use of elevated temperatures to enhance solubility is one useful approach. Here, the working fluid is heated prior to application of the electrical field; such a protocol was used to prepare a thirdgeneration ASD of helicid with PVP [100]. The elevated temperature does not have a significant influence on the PVP chain entanglement density, but can reduce the surface tension and viscosity of the working fluid while increasing the drug solubility.

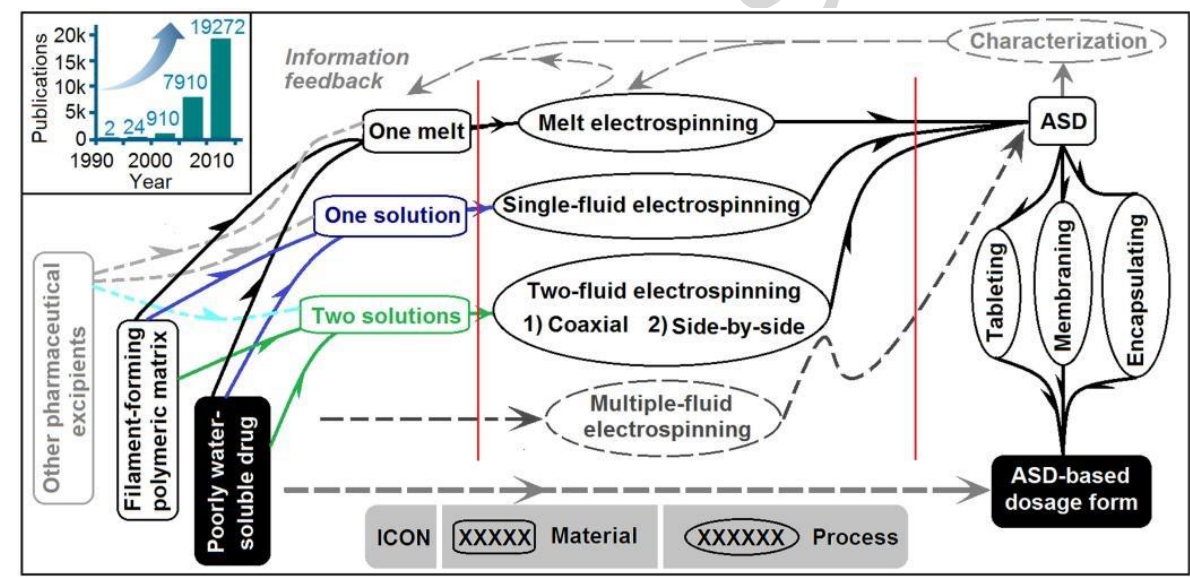

Fig. 3. Procedures for developing electrospun ASD-based dosage forms of poorly water-soluble drugs. The top-left inset shows the number of publications with "electrospinning" as the topic, as determined from a Web of Science search in five year intervals from 1991.

In some cases, a suitable solvent or a solvent mixture for electrospinning cannot be found. There is also a range of situations (e.g. with a heat-labile API) where melt or elevated temperature electrospinning is not appropriate. In these situations, doublefluid or even multiple-fluid electrospinning processes can be applied. These can be exploited not only to obtain ASDs that can enhance the dissolution rate but also to provide multiple-phase drug release profiles. For a two-fluid process, there are only two possible classes of products: those with a side-by-side configuration (a Janus structure) or an inner-outer arrangement (core-shell or core-sheath) [150,151]. These are respectively generated by side-by-side or coaxial electrospinning. The production of core-shell structures by coaxial electrospinning has been reported in a number of publications, with a focus on the preparation methods and/or materials with improved functional performance. In terms of performance, desirable goals may include 
protection of the core from the outside environment; preventing the core from aggregating; maintaining the material's compositional and structural integrity; or, selective percolation of molecules in and out of the interior of the material $[152,153]$.

The implementation of coaxial electrospinning is one of the major breakthroughs in this field [154]. It was thought for a long time that the shell fluid must be electrospinnable to enable fiber generation and produce a core-shell nanostructure [155]. However, a modified coaxial electrospinning process was recently reported where the shell fluid can be a dilute polymer solution, a salt or surfactant solution, or even a pure solvent [156-158]. This indicates that only one of the two fluids needs to be spinnable for successful coaxial spinning. This has made the coaxial process a powerful tool for formulating new types of structured ASDs which cannot be accessed in a single-fluid process.

As discussed above, there are two prerequisites for single-fluid spinning: codissolving of the drug and polymer in a solvent or solvent mixture, and obtaining a spinnable working solution. Both can be troublesome, and both can be overcome by using a coaxial process. For example, Li et al. reported the preparation of ASDs of quercetin using an electrospinnable shell solution (10\% (w/v) PVP and 0.5\% (w/v) SDS in a mixture of ethanol and water $(95: 5 \mathrm{v} / \mathrm{v}))$ to guide and encapsulate an unspinnable drug-loaded core fluid (10\% (w/v) PVP and 4\% (w/v) quercetin in a mixture of ethanol and DMAc (70:30 v/v)) [159]. Thus, it was possible to prepare an ASD of the PVP/quercetin core solution, which is not feasible in a single fluid process. In another example, $\mathrm{Wu}$ et al. reported a similar fast-dissolving ASD of helicid [146]. These examples exploit a spinnable sheath fluid to process an unspinnable core, but it is also possible to work with an unspinnable shell and a spinnable core. Such modified coaxial processes often use shell fluids to prevent clogging during the electrospinning processes [160-162].

To date, there are only a limited number of publications on side-by-side electrospinning, due to it being difficult to prevent the separation of the two working fluids during the process [150,163-166]. However, as is the case with coaxial electrospinning, only one of the two fluids needs to be electrospinnable to enable a successful side-by-side spinning process [167-170]. Janus nanofibers potentially have several advantages over core-shell structures. For example, interactions between the core and shell fluids might cause a coaxial process to fail, but this may not be a problem in side-by-side spinning because the contact area between the fluids is much smaller. In the applications of ASDs for controlled release, Janus fibers have both sides in contact with the environment, and thus can easily be designed to provide programmed controlled release of multiple components $[150,170]$.

One should note that it is impossible to consider the preparation of working solutions, the type of electrospinning process to be used, and the associated 
processing parameters as independent from one another. Implementation of a feedback process between the processing parameters and product characterisation ensure effective optimisation.

Nanofiber-based SDs can in some cases be regarded as finished products (e.g. for wound dressings). Their conversion to solid dosage forms for potential consumer products may however also require downstream processing methods, as addressed in several publications, using traditional pharmaceutical technologies [171]. Nanofiberbased ASDs can be easily cut into wafers [172] or formulated as tablets or capsules for oral administration [173], applied as patches for topical applications, or utilised as vaginal suppositories or drug eluting stents [142]. Nanofibers in the form of nonwoven fabrics have significant advantages in their ease of onward processing compared with nanoparticulate ASDs. This has been demonstrated for poly(vinyl pyrrolidone/vinyl acetate) (PVPVA) fibres loaded with the anti-fungal drug itraconazole created using a high-throughput corona electrospunning process with a conveyor-belt collector [171, 174].

\subsection{Advantages of electrospinning in creating SDs}

In a broad sense, the development of advanced materials is dependent on the technologies that are available to create them. Although the evolution between the different generations of SDs is, to some extent, based on the advent of new pharmaceutical carriers, the past half-century has seen only a limited number of new raw materials being introduced into SDs. For applications in improving the solubility and dissolution rate of poorly water-soluble APIs, these excipients are water-soluble polymers (PVP, PEG, PEO, PVA, cellulose and its derivatives, such as HPMC and HPC), inorganic particles $\left(\mathrm{SiO}_{2},\right)$, pH-sensitive polymers (e.g. Eudragit ${ }^{\circledR}$ E100, Eudragit $^{\circledR}$ L100 and HPMC-AS) or insoluble polymers (e.g. Eudragit RSPO, Eudragit RLPO and EC). However, new technologies are continuously emerging in the field of pharmaceutics and lead to new approaches for the generation of SDs using the same family of polymeric excipients.

One example of this evolution is the melt-based SD technology, which has developed from eutectic mixtures to co-fusion melts, then to the extrusion melt, and to two-screw extrusion methods. New techniques are often introduced to resolve the problems encountered in the previously utilised approaches, and to create new types of SDs with improved functional performance. A new technology will be adopted only if it has significant advantages over its counterparts and if its products are superior to those produced by other technologies, however [175]. Compared with traditional SD technologies, electrospinning and nanofiber-based SDs have a series of desirable properties as detailed below. 


\subsubsection{Technical advantages}

\section{(1) Extremely fast drying}

Dissolving both the poorly water-soluble APIs and the polymeric excipients in a common solvent or a mixed solvent system and then evaporating the solvent to obtain an ASD is a widely utilised process. Many different technologies have been developed to remove the solvents more quickly and completely, such heat drying, vacuum drying, using hot air or nitrogen, spray-drying, and using supercritical fluids [176-178]. Electrospinning is advantageous here because its evaporation rate is extremely fast, whereas with the other processes it is often very hard to remove all the organic solvents in a rapid manner. This in turn can result in the formation of drug crystals in the final SD $[65,179,180]$.

Since the solvent quickly evaporates in electrospinning as the fluid jets travel to the collector, the polymer filaments rapidly solidify, reducing the mobility of the drug molecules [181]. When the solvent is evaporated completely, the drug molecules are 'frozen' in the polymer fiber matrix in a random and highly homogeneous manner, which leaves little possibility for the drug molecules to move and come into contact to generate crystal lattices [149].

(2) High versatility

Although only around one hundred filament-forming polymers can be electrospun to form fibers, the electrospinning process has broad applications for creating ASDs of poorly water-soluble APIs. A wide range of APIs can be processed into drug-loaded nanofibers, even for fragile bioactive ingredients such as peptides and proteins $[182,183]$. All fluids used in traditional SD technologies, such as solution, melt and melt-solution processing, can be processed using electrostatic energy to prepare fiberbased SDs so long as they have appropriate viscosity. Further, there exist a variety of electrospinning processes, from which it is almost always possible to identify a suitable modality for a particular investigation. This is not easily achievable using conventional SD techniques that may have issues with the lack of a suitable solvent system or API temperature sensitivity [146,184]. Many drugs have $\mathrm{pH}$-dependent solubility [185,186], and structured nanofibers can be developed for preparing SDs as an acid-base pair using coaxial or side-by-side spinning.

Beyond these advantages, it is important to note that it is not only polymers, which can be used for making electrospun fibers. Small molecules with extensive intermolecular interactions such as $\beta$-cyclodextrins and phospholipids can also be processed [187-189]. Where APIs, such as proteins, are not compatible with an organic solvent they can be incorporated into the core of core-shell nanofibers using a water-based core fluid [190]. Finally, elevated temperature electrospinning can be 
exploited to prepare ASDs from aqueous solutions for those APIs only soluble in hot aqueous solutions $[100,191]$.

\section{(3) Facile product collection}

Electrospun nanofibers are most commonly assembled into random non-woven fabrics, which can be easily peeled away from the fiber collector. Although the individual fibers have diameters on the nanoscale, the mats are visible, meaning their further processing is facile. In comparison, the collection of nanoparticles is normally more challenging, as they are not interwoven in the same way that the fibers are. If desired, electrospun nanofibers can also be aligned or tailored to form macrostructures, such as multi-layered matrices [192,193], but relatively little work has been carried out in this regard.

\section{(4) Single-step nanofabrication}

All types of electrospinning are simple single-step processes. This one-step conversion from working solutions to solid fibers makes it attractive in the pharmaceutical arena. With some traditional ASD production methods the products are bulk solids, which need to be crushed and sieved for improved dissolution performance. Thus, they often involve multiple downstream processing steps, which are time-consuming and can result in poor reproducibility and robustness [194].

\section{(5) Energy-efficiency}

Compared with traditional SD methods that exploit thermal or mechanical energies, the electrospinning process directly uses electrical energy, omitting the conversion of electrical to other types of energy and the losses that always occur upon so doing. Thus, electrohydrodynamic atomisation processes not only provide more efficient drying but also have higher efficacy in their usage of energy.

Other advantages of the electrospinning process include its capability of generating complex nanostructures, which can easily be produced on a large scale. For example, multiple-jet electrospinning, edge electrospinning, corona-electrospinning and needless electrospinning have all been reported for scaling-up the fabrication of electrospun fibers [109,195-198].

\subsubsection{Product advantages}

(1) The ability to generate unique SDs 
The unique properties of electrospun fibers make them perfect composite materials to be utilised as SDs. The process is able to 'freeze' the guest drug molecules randomly in the filament-forming matrix, forming a state comparable to the liquid state but also propagating the structure of the spinneret to the solid product. Thus, in the structured fibers produced from double or multiple fluids, the components can also be localised at certain points in the fibers, because there is typically little diffusion from one fluid to another during the rapid drying process [199]. The electrospun fiber mats also have benefits in that: i) the fibers always possess a very small diameter and an extraordinary large surface area; ii) the assembled nanofiber fabrics have a 3-D continuous web structure with high porosity; iii) the mats often have excellent mechanical properties, high axial strength, and extreme flexibility. These characteristics mean that electrospun SDs can effectively facilitate the dissolution of poorly water-soluble drugs and benefit the development of solid dosage forms.

\section{(2) Tailoring functional performance}

Polymers are crucial in the development of novel DDSs [200, 201]. Electrospinning can provide a series of strategies to develop new polymer-based SDs with programmed functional performance. Electrospun nanofiber-based ASDs, particularly the second, third and fourth-generation products, are usually used to provide fast drug dissolution profiles, particularly for those APIs belonging to BCS Class II [202]. However, with the introduction of insoluble polymers in creating ASDs, fourthgeneration SD for achieving controlled release profiles have been reported. For instance, the first ever publication of electrospun drug-loaded fibers reports such systems [203]. Within the monolithic SDs from single-fluid spinning, other functional ingredients such as trans-membrane enhancers, sweeteners and surfactants can be incorporated for improved therapeutic effects and patient acceptability [149]. Compared with traditional pharmaceutical dosage forms such as tablets, the homogeneous or molecular distribution of APIs within the polymer matrices of fourth-generation CR-SD fibers can provide more accurate and robust controlled release profiles governed by the carriers' physio-chemical properties. However, a more appealing strategy is to combine fourth-generation SDs with second or thirdgeneration systems in a nanostructured manner (fifth-generation SD).

For example, fifth-generation SDs can provide a biphasic drug release profile (with both fast and sustained release phases) where a number of performance factors can be controlled in a highly tuneable manner. These factors include the amount of drug release occurring in the first and second phases [204], the drug release target sites [205], the release mechanism [206], and the release rates in the sustained release phase [207]. It is easy to conceive of a system where two poorly water-soluble APIs 
are incorporated into a single structural nanofiber-based SD, with each in its own spatial compartment and the two acting synergistically for an improved therapeutic effect [208,209].

\section{(3) Enhanced stability}

Stability of SDs, particularly ASDs, is a persistent issue in pharmaceutics. Biologic-based APIs such as enzymes, protein and peptides have major stability issues which arise during their formulation. It is interesting to find that these actives have shown great stability when processed by electrospinning, as demonstrated by a number of studies [210-213]. If an appropriate selection of the polymer carrier is made, electrospun ASDs have good storage stability, which has been demonstrated by several recent investigations $[65,145]$.

With regard to the stability of drug dosage forms, three aspects need to be considered: (i) the chemical stability of the API during the formulation process, (ii) the API's physical stability during long-time storage, and (iii) the physiological stability of the formulation during application. Of these, the physical stability of ASDs during storage has drawn the most attention because the amorphous drug in an ASD is thermodynamically unstable, and will over time relax to a form a crystalline material. Lopez et al. investigated the stability of electrospun ASDs of indomethacin and griseofulvin using X-ray diffraction and differential scanning calorimetry (DSC), and their results demonstrated that both APIs were found to be stable and amorphous after 8 months' storage in a desiccator (relative humidity $<25 \%$ ) [145]. Démuth et al. have reported that the amorphous nature of itraconazole in electrospun hydroxypropyl methylcellulose (HPMC) nanofibers is maintained for up to one year, even at $40^{\circ} \mathrm{C} / 75 \%$ relative humidity [65].

Electrospinning also has great potential in stabilising ASDs through the intelligent design of the formulation. For example, the shell parts of core-shell fibers can be designed to act as a protective layer to prevent amorphous drug in the core from recrystallisation by isolating it from the surroundings, particularly humidity. Generally, the higher the glass transition temperature $\left(\mathrm{T}_{\mathrm{g}}\right)$ of the ASD, the lower the molecular mobility of the API, and thus the better the physical stability of the ASD. $\mathrm{T}_{\mathrm{g}}$ can be lowered by the presence of water [214], and thus a shell offering isolation from environmental humidity and a 3D web structure should increase the stability of core ASDs.

The advantages of electrospinning processes and their products over competing ASD generation methods should promote the development of commercial electrospun ASDs in the near future, particularly with the usage of high-speed electrospinning. There are some limitations presently associated with this technology that need to be carefully considered, such as the use of large amounts of organic solvents and the 
reproducible creation of structured fibers on the large scale. However, there are many reports of the high-speed creation of monolithic nanofibers [195-198], and thus it is clear that with time the necessary technologies will become available.

\section{A survey of electrospun fiber-based ASDs}

The term solid dispersion was defined in the early 1970s by Chiou and Riegelman as a dispersion of an API in an inert carrier in the solid state, prepared by either solvent, melting or solvent-melting methods [215,216]. Hence, in a general sense, all electrospun drug-loaded nanofibers can be classified as SDs, with a majority of them being ASDs. As discussed above, the original driving force for producing SDs was to improve the solubility and dissolution rates of poorly water-soluble APIs (the first to third-generation SDs). With the development of SDs, their functions have been extended to controlled release in fourth-generation SDs [18, 217]. More importantly, electrospinning has improved capabilities to create different types of complex nanostructures, and thus fifth-generation SDs can be prepared which cannot be realised by most traditional SD methods. The different categories of electrospun ASDs are illustrated in Figure 4, classified according to their distribution of drug molecules and other functional components. Some typical ASDs for enhancing dissolution rate are summarised in Table 2.

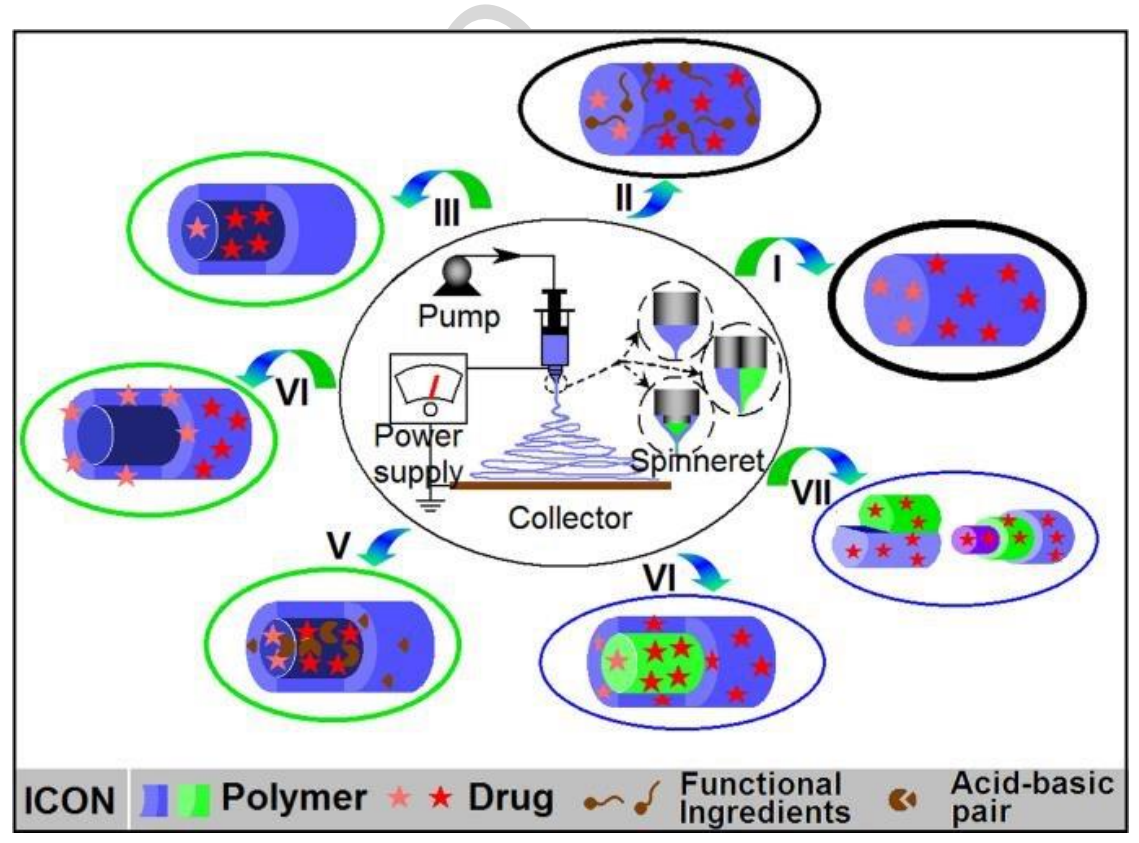

Fig. 4. Electrospun ASDs with different distributions of drug molecules and functional excipients: I) homogeneous distribution of drug within the polymer matrix; II) homogeneous co-distribution of drug with another functional ingredient; III) drug present only within the core of core-sheath fibers; IV) drug distribution only within 
the sheath of core-sheath fibers; V) distribution of a drug acid-base pair within the core of core-sheath fibers; VI) drug present both in the core and sheath of the fibers; VII) complex nanostructures with varied distribution patterns.

\subsection{Electrospun second-generation SDs}

The functionality of electrospun fibers is mainly dependent on the API and other additives that are loaded into the polymer matrix, the distribution of these ingredients in the fibers, and the interactions between the API, the polymer, and any other additives. For biomedical applications, the API can comprise small molecules, biomacromolecules, emulsions, suspensions, nanoparticles and even cells [218-220]. The most explored type of APIs are the poorly water-soluble drugs, most of which are small molecules [221].

For simple binary electrospun nanofibers, the therapeutic effect is determined by the incorporated drug, but the functional performance is governed to a large extent by the polymer matrix, just as with numerous polymeric DDS prepared using traditional pharmaceutical technologies. For dissolution enhancement of poorly water-soluble APIs, binary nanofibers are prepared composed of a drug in a hydrophilic polymer. Such materials comprise the majority of reported electrospun ASDs [11,63-65,93, $100,101,108,109,118,145,148,172,173,222,223]$. These ASDs are denoted as type I in Figure 4. The drug molecules are homogeneously distributed throughout the fibers, mirroring their distribution in the co-dissolving solutions used for spinning. These monolithic two-component nanofibers have received the most attention because of their ease of preparation. Some typical publications are listed in Table 2. These ASDs are mainly created using single-fluid solvent electrospinning, but can also be produced using melt electrospinning [93,148]. Although there are no reports of these yet, second-generation ASDs could also be fabricated using modified coaxial electrospinning (in which an organic solvent is used as the shell fluid to facilitate the electrospinning process and create high quality nanofibers) [115].

Using itraconazole as a model drug and hydroxylpropylmethyl cellulose as a filament-forming matrix, Verreck et al. were the first to intentionally prepare an electrospun fiber-based ASD to improve drug dissolution behaviour [64]. In other work, Yu and co-workers prepared ibuprofen-loaded polyvinylpyrrolidone (PVP) nanofiber mats, in which the drug could dissolve simultaneously with PVP, with complete dissolution reached within only 8.1s [224]. Later, the same authors compared SDs of acetaminophen/PVP prepared using different processes, and demonstrated that electrospun nanofiber-based second-generation SDs showed markedly better dissolution enhancement than other systems such as cast-film SDs, and films created using vacuum drying and freeze drying processes [63]. Nagy et al. 
reported ASDs prepared by melt electrospinning and compared them with analogous products from twin-screw hot melt extrusion, demonstrating that the electrospun $2^{\text {nd }}$ generation SD showed better performance [93]. Using hydroxypropyl cellulose (HPC), polyvinyl alcohol (PVA), and polyethylenoxide (PEO) and diosmin as model drug, Vrbata et al. investigated the preparation of binary ASDs using the Nanospider ${ }^{\mathrm{TM}}$ technology, an industrial scale electrospinning method based on the concept of needless electrospinning, suggesting the possible production of $2^{\text {nd }}$ generation SDs on a large scale [225, 226].

In terms of polymer selection, almost all polymers used in pharmaceutics or as edible coatings for food products can be used for electrospinning, provided that the drug and the polymer share a solvent or solvent mixture. Some of these polymers include PVP [224], PEO [227], PVA [11], HPMC [64], the Eudragit series [228] and also natural polymers such as gelatin [206]. In addition, other water soluble pharmaceutical excipients such as $\beta$-cyclodextrin and its derivatives can also be used to prepare second-generation ASDs [229]. 
Table 2. Some typical examples of electrospun ASDs used to accelerate the dissolution of poorly water soluble APIs.

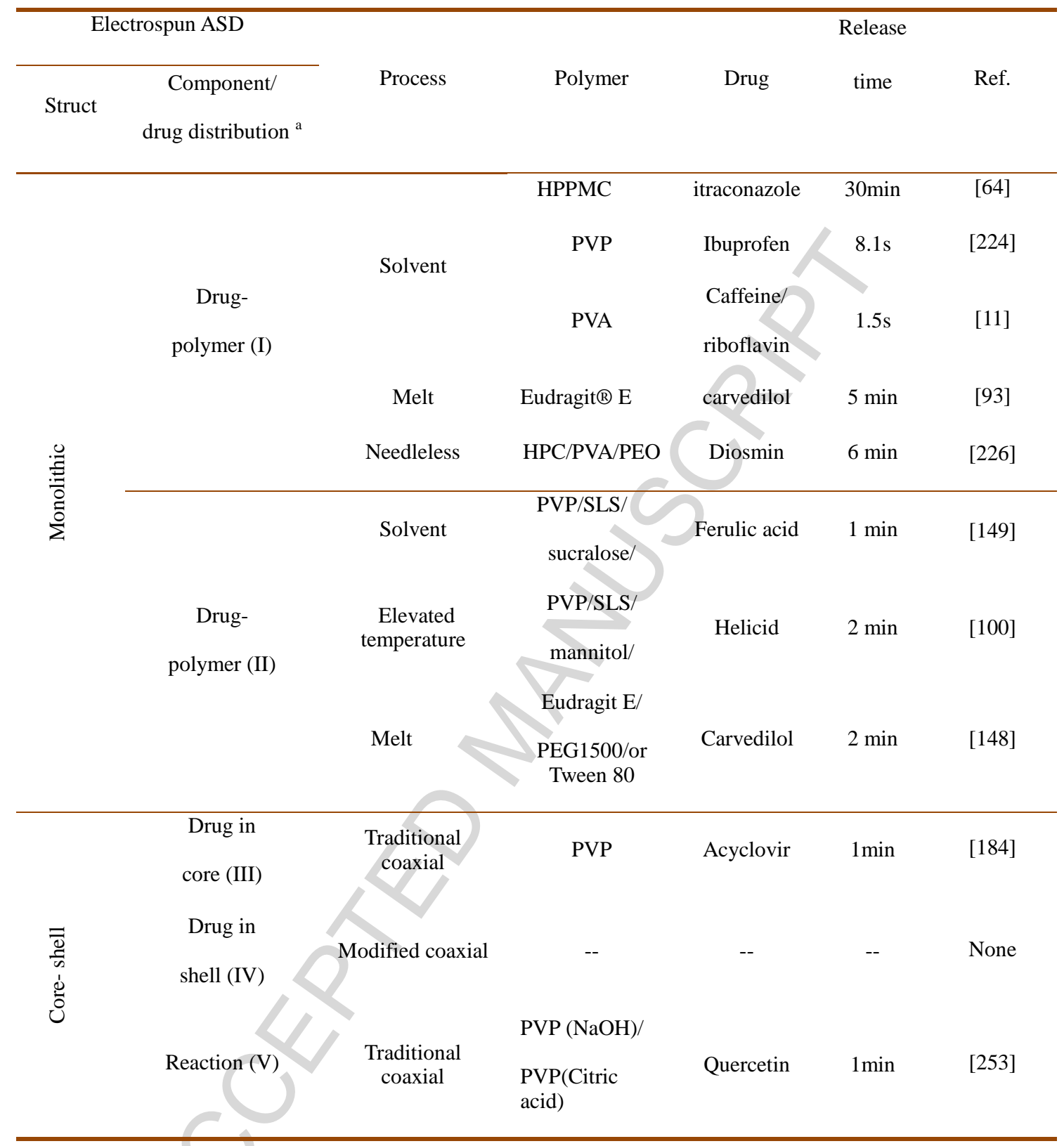

${ }^{a}$ The corresponding diagrams showing the arrangements of drug and polymer are shown in Figure 4.

\subsection{Electrospun third-generation SDs}


The third-generation of ASDs is different from the second in that they contain further additives that can promote dissolution through synergistic action with the polymer carrier $[17,45]$. Provided the drug, other component(s), and filament-forming polymer can be dissolved together to yield an electrospinnable solution, these ASDs can be similarly prepared by single fluid electrospinning. The addition of small amounts of surfactants or emulsifiers typically has very limited influence on the spinnability of polymer solutions. Thus, the electrospinning process should similarly propagate a homogeneous distribution of three or four components in solution to fabricate monolithic nanofibers with both the drug and other functional additives uniformly distributed throughout, classified as type II in Figure 4.

Such third-generation ASDs (e.g. one consisting of ferulic acid as a model drug, PVP, the surfactant sodium dodecyl sulfate (SDS), and sucralose as a sweetener) can been prepared using single-fluid electrospinning [149]. The third-generation ASDs have generally shown improved functional performance over analogous cast films and the raw drug particles both in in vitro dissolution and also in permeation through the sublingual mucosa. A third-generation ASD composed of helicid, SDS, mannitol and PVP was created using an elevated temperature electrospinning process with water as the solvent [100]. The presence of both SDS and PVP synergistically increased the wettability and saturation solubility of helicid. The nanoscale diameters and 3D web structure of the fibers facilitated the mass transfer of solvent and helicid molecules. All these factors worked together to give significantly increased dissolution and permeation rates, allowing a faster onset of action .

As a result of the electrospinnability of hydrophilic polymer excipients and the existence of secondary interactions between the functional components (drug, surfactant, transdermal or transmembrane enhancer, sweetener, and $\mathrm{pH}$ modifiers), the majority of third-generation electrospun SDs can be fabricated using solvent electrospinning. In a recent publication, however, Balogh et al. [148] investigated the feasibility of applying melt electrospinning to create third-generation ASDs. In addition to the drug carvedilol and polymer Eudragit E, a third component (triacetin, Tween ${ }^{\circledR} 80$, or PEG 1500) was added as a plasticizer, which in a melt processes effectively lowers the working temperature, helping to prevent thermal degradation of the drug. In vitro dissolution tests indicated that the addition of plasticizers could accelerate drug dissolution.

Further developments in third-generation electrospun SDs include new types of molecular self-assembly or self-emulsification templates. These systems are formed from fast-dissolving polymers, hydrophobic drugs and other hydrophobic agents. They exploit secondary interactions (such as hydrophobic interactions and hydrogen bonding) between the drug and other ingredients within the polymer matrix. The assembling components can be mixed at the molecular scale in a monolithic fiber or 
compartmentalised. When the fibers come into contact with water, the insoluble components they contain will act as building blocks and self-aggregate into zerodimensional nanoparticles or liposomes. The fundamental forces at work here are molecular recognition among the building blocks, and their hydrophobic natures proving a thermodynamic impetus to aggregate away from the aqueous environment. During this "bottom-up" process, the fibers act as templates, with the polymer chains providing steric confinement [230,231].

\subsection{Electrospun fourth-generation SDs (CR-SDs)}

Many electrospun drug-loaded fibers prepared from water insoluble polymers can provide extended release profiles and are essentially fourth-generation ASDs $[232,233]$. This is because the fibers prepared from a single co-dissolving working solution are monolithic nanocomposites, with the drug molecules uniformly distributed within the polymer matrix. Compared with standard tablets, the amorphous state of the drug and its highly uniform distribution in the fiber should ensure the drug release behaviour is controlled by diffusion, and if highly homogeneous systems are produced the release mechanism should be very reproducible and easy to predict.

In electrospun CR-SDs, the drug distribution is the same as in the second and thirdgeneration SDs (type I and II in Figure 4). Because of the use of water-insoluble polymers as the carrier, these ASDs release the loaded APIs gradually. To manipulate the drug release rate or duration, a third component which can act as a pore-forming agent to promote the diffusion of water and drug molecules can be added into the nanofibers [234,235].

Yan et al. reported the preparation of ferulic acid (FA)-loaded cellulose acetate (CA) nanofibers, in which PVP was included to act as a porogen and give an improved release profile [234]. The ternary nanofibers exhibited better release profiles than fibers of FA and CA alone in terms of release completeness, reduced tailing-off, and adjustable release rates. Similarly, Qian et al. reported fourthgeneration CR-ASDs comprising ibuprofen/polyacrylonitrile (PAN) nanofibers. Triple-component systems additionally containing PVP could release $97.8 \%$ of the embedded ibuprofen, significantly higher than the $73.4 \%$ freed from binary ibuprofen/PAN fibers. The inclusion of PVP was able to effectively preclude the entrapment of drug molecules in the insoluble PAN matrix [235].

When using fourth-generation ASDs for sustained release, an initial burst of release is inevitable despite the insoluble nature of the fibers. Their large surface area and the amorphous physical form of the drug results in a significant amount of drug being present at the fiber surface, which is rapidly released upon contact with a liquid 
medium [236, 237]. A blank polymer coating on such fourth-generation ASDs can help to prevent this initial burst release, and such a system can be realised easily using coaxial electrospinning. For instance, a blank coating of cellulose acetate (CA) on ketoprofen (KET)-loaded CA nanofibers led to a zero-order drug release profile over $96 \mathrm{~h}$ without any initial burst release [238]. Similarly, a blank zein coating on KETloaded zein fibers could enable linear drug release over $16 \mathrm{~h}$ [239]. These ASDs can provide programmed drug release profiles due to their specific structural characteristics; this is impossible using traditional monolithic ASDs. They have a core-sheath structure with drug present only in the core, and are thus fifth-generation SDs: type III in Figure 4.

\subsection{Electrospun fifth-generation SDs}

The fabrication of advanced DDSs is dependent increasingly on controlling the structure-property-function relationship at the nanoscale [240,241]. Core-shell nanostructures have received by far the most attention in the development of nanoscale DDSs, with much less effort invested in exploring other nanostructures such as Janus, tri-layer and multiple-compartment architectures [242-245]. In the examples above, the shell is used simply to prevent an initial burst of release $[238,239]$. However, both the core and shell can be loaded with APIs or other pharmaceutical excipients. The core-shell structure has been broadly used in attempts to overcome the challenges faced in areas such as the controlled release of multiple APIs from one dosage form, multiple phase controlled release, and to ameliorate concerns associated with multiple drug resistance [204,246,247].

As shown in Figure 4, systems of type III, IV and V are typical fifth-generation SDs used to provide improved solubility or dissolution rate of APIs, with the drug present only in one part of the core-shell nanofibers.

Such systems are often prepared where it is impossible to prepare an electrospinnable co-dissolving solution of drug and polymer. For example, core-shell type III SDs of acyclovir (ACY) have been prepared using a spinnable shell fluid to guide and surround a core un-spinnable fluid. Acyclovir has a high melting point of $257^{\circ} \mathrm{C}$ and also has poor solubility in the organic solvents such as methanol, ethanol, acetone and chloroform commonly used for electrospinning [184]. Few publications can be found on acyclovir ASD products prepared using traditional melt and solvent evaporation approaches. ACY is soluble in DMAc, but PVP cannot be electrospun using this as solvent due to its high boiling point $\left(166^{\circ} \mathrm{C}\right)$ and low volatility. Thus, it is very difficult to prepare nanofiber SDs of ACY through a single fluid electrospinning experiment. The use of coaxial electrospinning can resolve this problem easily through the use of two fluids, with only one of them (in this case the 
PVP shell) needing to be spinnable. The resultant fibers were found to release the loaded ACY within 1 minute and provide a 6-fold increase in the permeation rate across the sublingual mucosa (compared to crude ACY particles) [184].

The encapsulation of APIs in the core of a fiber not only can provide enhanced dissolution performance, but also can improve the stability of the amorphous composites in the core, with the shell acting as a barrier to environmental humidity. This type of SD may also increase the API's chemical stability through isolating the core from contact with environmental light, heat, and oxygen .

To date, there are no reports of type IV SDs with the API only distributed on the surface of the fiber. However, this structure is potentially very useful for promoting the dissolution of poorly water-soluble APIs. In traditional electrospinning, the fibers commonly have diameters in the range of $100 \mathrm{~nm}$ to $1000 \mathrm{~nm}$. Sizes of $50 \mathrm{~nm}$ are very difficult to create by the electrospinning processes directly [248], but it is very easy to create nanofibers with a shell thickness of less than $50 \mathrm{~nm}$. The modified coaxial electrospinning processes in particular offers a series of protocols to give such narrow shells [238, 239]. For example, the concentration of solutes in the shell fluid, the flow rate and the shell-to-core fluid flow rate ratio can be controlled to manipulate the thickness of the fiber shell. Thus, fibers with drug only in the sheath compartment are anticipated to give exceptionally fast dissolution rates.

It is reported that two-thirds of poorly water-soluble APIs have pH-dependent solubility [17, 54]. Correspondingly, many $\mathrm{pH}$ modifiers have been added ton SDs, with the aim of improving the dissolution of such APIs [249-251]. Based on the coresheath structure, new strategies can be developed for creating SDs with different local environments for the API and charge neutralizers. The type V systems in Figure 4 comprise typical structural fifth-generation ASDs, with an acid-base pair loaded in them to permit the fast dissolution of poorly water-soluble APIs yet also give a neutral bulk solution. In a recent study, quercetin was explored. This is a flavonoid investigated for treating a range of different diseases such as high cholesterol levels, heart disease, diabetes and cancer. It is an acidic drug so its solubility increases with $\mathrm{pH}$ [252]. A co-dissolving solution of quercetin, PVP and sodium hydroxide was used as the core fluid, and a spinnable solution of citric acid and PVP as the shell. In vitro dissolution tests demonstrated that the drug can be released immediately when the fibres are exposed to an aqueous solution, and release did not change the bulk $\mathrm{pH}$ value of the medium because of to the presence of the acid-base pair [253]. This finding opens a new route for developing ASDs of poorly water-soluble APIs with $\mathrm{pH}$-dependent solubility using electrospun polymeric nanostructures.

Type VI and VII systems (see Figure 4) provide programmable release profiles through their multiple-compartment structures and will be discussed later. 


\section{Evaluation of electrospun SDs}

\subsection{Characterisation of electrospun nanofiber-based ASDs}

The routine characterisation of electrospun systems has two aspects: an assessment of their morphology and structures, and their role as SDs (including the drug distribution and physical form, its compatibility with the polymer matrix, and the SD functional performance). The morphology and internal structure of the fibers can be characterised using a series of microscopy techniques including optical, polarised light, fluorescence, laser scanning confocal, scanning electron (SEM) and transmission electron (TEM) microscopies [158,254,255]. Image analysis can be undertaken using software such as ImageJ to evaluate the nanofibers' diameter and size distributions, typically based on SEM images [256,257]. The physical form of the components and their compatibility can be determined using XRD, DSC, Raman and infrared spectroscopies, all of which will be familiar to researchers in this field. The interested reader is directed to some excellent reviews which have documented recent progress in these techniques [258,259]. Other important characterisation includes a determination of the drug encapsulation efficiency of the fibers (which is often almost $100 \%$ and can be determined using UV-vis spectroscopy, high performance liquid chromatography (HPLC) or other quantitative analysis methods) and the stability of the API after electrospinning (which is often explored using HPLC or nuclear magnetic resonance spectroscopy) [260,261].

The stability of electrospun ASDs on storage, their downstream processability and functional performance (taking into account the API's solubility and dissolution behaviour, the desired release profile, permeation effectiveness, clinical applications and bioavailability) must all be robustly assessed. Because the conversion from the as-formed nanofiber-based ASD to a commercial solid dosage forms is expected to have minimal impact on the ASDs, almost all the characterisations in the literature are on the electrospun fibers themselves. From this, useful data can be obtained to inform the preparation of working fluids and the processing parameters used for electrospinning. However, further exploration of the fibres post-processing will be required before they can be developed into commercial formulations.

Appropriate mechanical properties are important in the preparation of SDs based on tablets for oral application. This is because the downstream processing needs to be robust, reproducible and continuous [89]. Powder flowability and workability are key parameters for the conversion of nanoparticle-based SDs to commercial solid dosage form [262,263], and can often be problematic. However, electrospun nanofiber-based SDs could easily be transferred into capsules for commercial applications (Figure 3), 
and there are also reports of post-processing of the fibers to produce fragments which can be incorporated into tablets [171].

\subsection{Stability}

Similarly to ASDs fabricated using traditional methods, the stability of electrospun ASDs is a major concern. DSC (including modulated temperature DSC) and XRD are the most common methods used to detect crystalline material in the ASDs. DSC is able to detect crystallinity only where at least $2 \%$ of the material is crystalline however, and XRD struggles to detect crystalline material at below 5-10\% [258, 264]. More sensitive methods to detect the beginning of drug recrystallisation and to monitor the growth of drug crystals in their ASDs are thus highly desired [265]. A series of new protocols has been investigated to this end [266-270]. The Nagy group recently investigated the applications of some of these methods to monitor drug recrystallisation in electrospun ASDs and to determine their long-term stability. The formulations were stored under controlled conditions (following the International Conference on Harmonisation guidelines) and Raman microspectrometry was applied to identify traces of crystalline itraconazole in aged drug-PVPVA ASDs after one year at $40^{\circ} \mathrm{C} / 75 \%$ relative humidity. A multivariate curve resolution-alternating least squares method was exploited to obtain a reference for amorphous itraconazole in the matrices, and on this basis the amorphous/crystalline ratio was determined [65].

Electrospun nanofiber-based ASDs typically have good physical stability because the drug tends to be molecularly dispersed in the fibers, and the polymer chains provide steric hindrance to drug recrystallisation. With careful selection of the polymer carrier to ensure that favourable secondary interactions exist between the drug and polymer, the API can be anchored to the polymer through $\mathrm{H}$-bonding and similar interactions, further restricting movement. A diagram considering the drug's physical form, the possible phase separations which may arise (A, B and C in I, II and III, respectively), and a method for improving the physical stability of electrospun ASDs (from I to IV) is given in Figure 5.

Type I represents a case where the drug and the polymer have minimal secondary interactions (such as hydrogen bonding, hydrophobic interactions and electrostatic interactions) existing between them, and where the drug molecules are distributed among polymeric molecules and isolated from one another. An example of this is itraconazole with PVPVA, an ASD where there are only weak hydrophobic interactions between the benzene rings of itraconazole and the carbon chains of PVPVA [65]. Type II represents a situation in which the drug and the polymer undergo some intermolecular interactions, and the drug molecules are thus associated 
with the polymer chains. One example is PAN with ACY: the former can act as a proton receptor and the latter as a proton donor [261]. Type III represents the most favourable case in the design of ASDs: here, the drug and the polymer molecules have strong interactions with one another. These interactions should ensure long-term stability of the ASDs because the drug and polymer molecules are tightly bound together. For example, when using zein molecules with ferulic acid, the two species can each act as both proton donors and acceptors for forming hydrogen bonds [271]. More details of the molecular interactions between drug and polymer can be explored through molecular modelling simulations using software such as HyperChem or ChemBio3D [145].

The stronger the interactions between the drug and polymer molecules, the better the uniformity and stability of the ASDs is likely to be, and the less the propensity for solid phase separation and API re-crystallisation to occur during fiber preparation and storage. This is because to recrystallize, the drug molecules must undergo four steps: translation within the solid matrices; coming into contact with other drug molecules; forming a crystal nucleus; and, crystal growth. These processes, especially the molecular mobility of drug molecules, can be prevented by the polymer. Particularly when in the brittle state, the polymer chains are able to lower molecular mobility and form a barrier to the movement of drug molecules. Thus, the higher the $\mathrm{T}_{\mathrm{g}}$, the better the physical stability of the ASD. The adsorption of moisture in an ASD can lower its $\mathrm{T}_{\mathrm{g}}$ value and thus reduce stability [272]: as a result, high levels of humidity should be avoided during production, downstream processing and storage of ASDs.

Considering the three cases (I, II and III) discussed above, case I relies mainly on the polymer having a high $T_{g}$ value to impart stability. In contrast, with the interactions between drug and polymer in cases II and III, the steric barrier to movement is supplemented with other forces. It is to be expected though that the stronger the interactions between drug and polymer, the greater the effect the addition of drug will have on the spinnability of polymer solutions, however. The drug can exert an influence on the surface tension and electrical conductivity of the working solutions, and also the physical entanglements of polymer molecules. Happily, this influence is often sufficiently small that there is no reduction in the spinnability of the co-dissolving fluids. In type III systems, the drug molecules may directly contribute to the formation of fibers during the electrospinning processes, bridging between adjacent polymer molecules and acting in a complementary manner to the physical entanglements [273].

Besides increasing the stability of ASDs, favourable interactions between drug and polymer should also elevate the drug loading capability. Thus, case III systems should allow the largest drug loading. If excessive drug (i.e. a greater amount than the drug's solubility in the polymer) is encapsulated in the ASDs, then phase separations can 
occur, marked "A", "B" and " $\mathrm{C}$ " in Figure 5. "A" represents API crystals which have formed because of a lack of strong drug-polymer interactions. However, in the cases "B" and "C", solid phase separation may not mean drug re-crystallisation. A degree of phase separation might arise with the fiber components remaining in the amorphous state, for instance with some areas having with a larger content of drug molecules than others. For instance, in an electrospun helicid-loaded ASD, phase separation was observed by TEM, but DSC and XRD did not detect any crystalline drug. This can be attributed to the existence of favourable drug-carrier interactions [100].

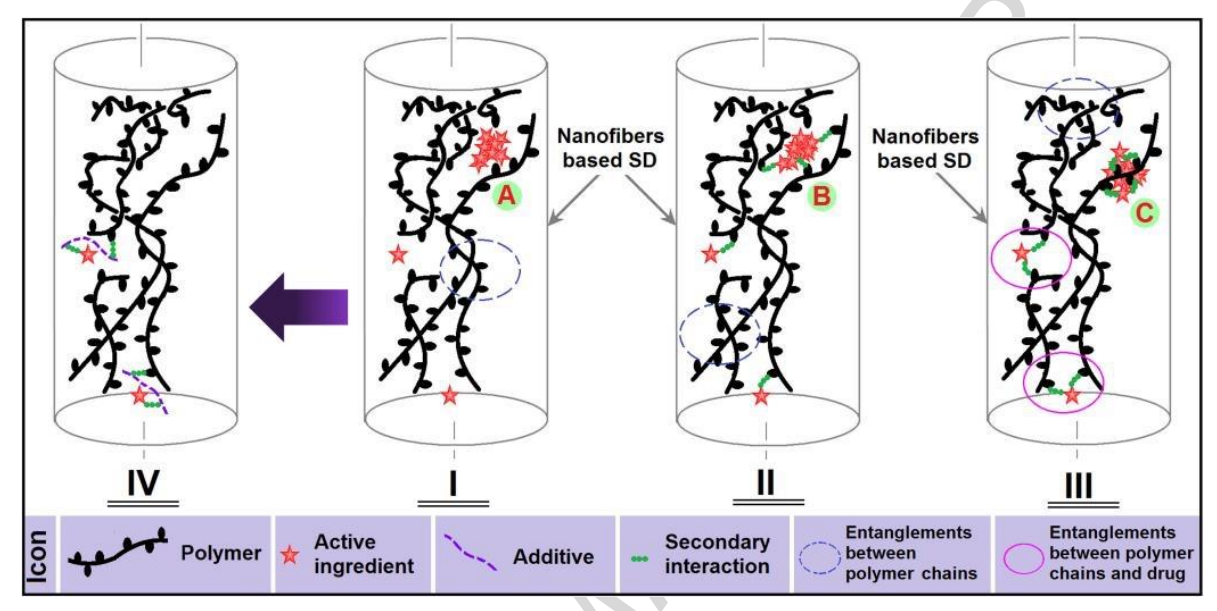

Fig. 5. The possible physical states of drug molecules in ASDs, and the implications for their stability.

In order to increase the interactions between the drug and carrier, a third or even a fourth excipient can be added to binary systems. As shown in Figure 5, a third additive may bridge the drug and polymer molecules and form secondary interactions with both of them. During electrospinning, the polymer leads to the formation of nanofibers, while the additive acts as an inhibitor to prevent crystallisation. For example, in binary PVP and itraconazole systems, excipients such as mannitol and polyethyle glycol (PEG) can be added to improve the molecular interactions and form an ASD with improved stability. The PEG can form hydrogen bonds with both the polymer matrix and the drug molecules. A smaller fiber diameter may also promote the stability of ASDs.

\subsection{Functional performance quantification}

Although the quality of nanofiber-based ASDs is commonly comprehensively described, most publications evaluate functional performance simply using in vitro dissolution tests. Some literature goes further to carry out in vitro permeation studies 
$[100,149]$. To develop electrospun ASDs into commercial products, there remains the need to perform toxicology studies, in vivo experiments, preclinical and clinical studies. The methods required to undertake these more advanced evaluations already exist and are widely used with traditional ASDs. Vrbata et al. recently reported electrospun ASDs of diosmin with a combination of HPC, PVA and PEO. Compared to a micronised crystalline form of the drug, an enormous increase in the dissolution rate was found with the ASDs. This resulted in a higher initial drug plasma level and higher AUC values after oral administration of the electrospun ASDs to male and female Wistar rats, suggesting greater in vivo bioavailability and also a faster therapeutic effect [226].

Since the filament-forming polymers employed in electrospinning have already been intensively exploited in pharmaceutics (e.g. PVP, PEO, Eudragit, HPMC and cellulose derivatives), they have typically been approved by the FDA and are "generally regarded as safe" excipients. In electrospun fibers, the organic residues are thought to be virtually completely removed (and the fibers can be kept under vacuum or in a desiccator to exhaust any remaining organic solvents if required) [274]. Thus, it is often thought that toxicology studies are not an important issue at this stage of development, since the safety of the fiber components has been well established.

Electrospun ASDs composed of water-soluble polymers provide rapid release where the drug dissolves and diffuses in the dissolution media on an extremely rapid timescale, and where it can be problematic to quantify release (Table 2). A series of simple methods has been put forward to record the dissolution of electrospun ASDs. Particularly for those ASDs intended for buccal or sublingual drug delivery (where typical dissolution tests designed to mimic passage through the gastrointestinal tract are not appropriate), methods have been designed for detecting dissolution using only small amounts of dissolution media, expected to mimic the local bio-membrane conditions $[11,275]$.

The initial goal of SDs was to improve the solubility and dissolution rates of poorly water-soluble APIs. However, as their development has proceeded, these aims have broadened to encompass almost all types of drug release profiles, such as immediate release, sustained release (fourth-generation SDs), pulsatile release and multiplephase release (fifth-generation SDs) [127,128,204-207]. These drug release profiles can be assessed using traditional in vitro dissolution tests according to the US or European pharmacopoeias. The release profiles obtainable from electrospun ASDs with nanostructured architectures will be discussed later.

To reach the systemic circulation, oral drug delivery systems must undergo two subsequent processes: firstly the drug disperses/dissolves in the physiological fluids, which requires a DDS with some hydrophilic properties, and later the drug molecules must pass through lipophilic bio-membranes, which demands some lipophilicity. 
Thus, ex vivo permeation tests are vital to fully characterize their performance. Class II APIs tend to have some lipophilic properties, and their onset of action of can be enhanced with very rapid release because of the large concentration gradient which results [276]. The incorporation of a lipidic carrier into fibers can also be used to accelerate permeation [277].

\subsection{Mechanisms underlying the enhanced dissolution rates of electrospun ASDs}

The mechanism of drug release from ASDs is a much debated. For traditional bulk ASDs, any improvement of dissolution rate for poorly water-soluble drugs is mainly dependent on the existence of the amorphous physical form of the API in the formulation and the water solubility of the carrier. For ASDs in the form of nanoparticles, the large surface area, API amorphous physical form, and the properties of the carrier all contribute to promoting rapid dissolution [278]. Compared to nanoparticle-based ASDs, nanofiber systems have additional physical properties to further enhance drug dissolution rates in aqueous media because of their continuous 3D web structure and its high porosity (Figure 6a).

It is well known that the dissolution of a polymer often involves several sequential steps: absorbing water, swelling, disentanglement, and finally dissolving into the bulk medium. For bulk ASDs water molecules need a long time to diffuse into the inner part of the formulation, which often occurs layer-by-layer via an erosion mechanism. When nanoparticles are placed into water they often aggregate [279]. These factors can slow the dissolution of APIs from bulk and nanoparticle-based ASDs. When nanofiber-based ASDs are placed into water, some small pores in the mat may disappear as the fibers take up water and swell (indicated by "A" in Figure 6a). However, the pores in the mats often have sizes much larger than the fiber diameters, with the mats having a porosity of around 80-90\% [280]: thus most of the pores will remain open even after swelling.

The mechanisms through which the dissolution rate is enhanced with electrospun ASDs can include the following: 1) the web structure makes it easy for dissolved drug molecules to diffuse from the fiber mat to the bulk media; 2) this means it is possible to maintain a relatively high drug concentration within the fiber mat (greater than that in the surroundings), which provides an osmotic gradient and promotes diffusion away from the mat; 3 ) the high surface area leads to a thin boundary layer (a small value of $\delta$ in Equation 1), in contrast to nanoparticles which easily aggregate; 4) within the fiber mat and its locality, there is a high local concentration of polymer, which hinders precipitation and crystallisation of the API during the dissolution process, permitting local supersaturation [281, 282].

The amorphous state of the API within the fibers and the homogeneous distribution 
of drug molecules throughout the matrix are also key to enhancing the dissolution rate (Figure 6b), just as with ASDs prepared using traditional pharmaceutical techniques. A number of factors differentiate the nanofiber-based ASDs from crude drug particles in terms of their release mechanisms. During the swelling of the fibers, the absorbed water molecules can easily replace the drug molecules within the polymer matrix and free them into the dissolution medium. The amorphous physical form of the drug in the fibers means there is no lattice energy barrier to dissolution. The fibers have huge surface areas and web structures, leading to a very large contact area with the dissolution medium, while in sharp contrast crystalline drug particles are dissolved through erosion of drug molecules from the crystal surfaces, with only a limited contact area existing between the drug and the liquid. Further, the properties of nanofiber-based ASDs can be easily tailored to allow them to further enhance the dissolution rate, for instance through the combined use of polymer matrices and surfactants. The latter allows the surface tension to be effectively reduced while hydrophilicity and wettability can be substantially improved.

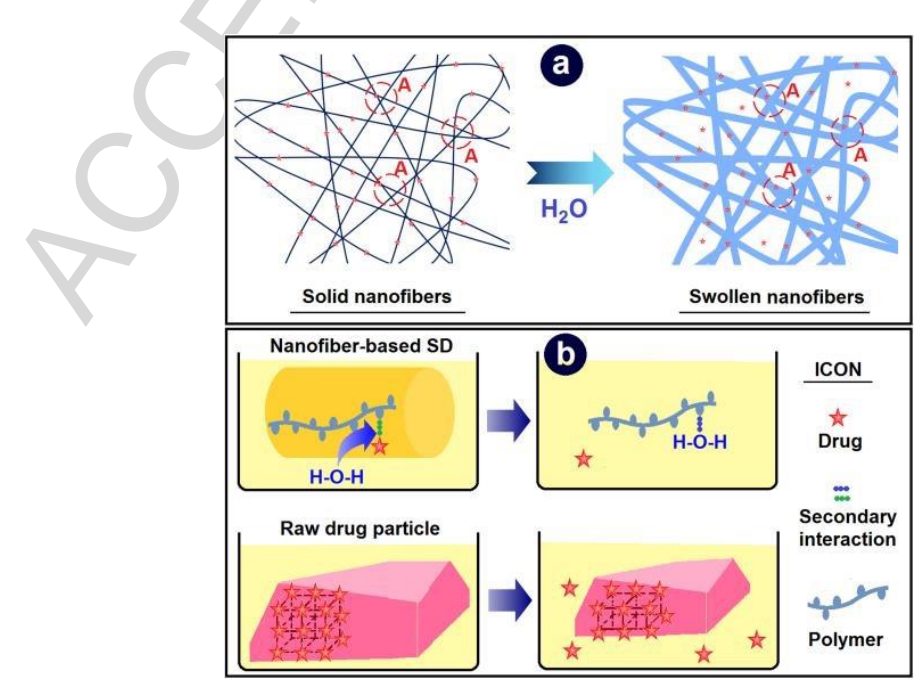

Fig. 6. The mechanisms by which electrospun ASDs can accelerate dissolution. (a) 
The 3D web structure remains porous even after the fibers have absorbed water and swollen; (b) Compared with raw drug particles, nanofiber-based ASDs have a series of properties leading to much more rapid dissolution of incorporated drug molecules.

\subsection{Drug dissolution profiles from different types of electrospun ASDs}

In the broad sense of being systems with "drug dispersed in a solid matrix" [283], all drug-loaded electrospun nanofibers can be considered SDs. Nearly all electrospun fibers are also amorphous SDs, in which the drug molecules are dispersed into the polymer matrix with little phase separation. As is discussed above, the second to third-generation electrospun SDs can release all the loaded API within a few minutes. In the literature, almost all in vitro dissolution tests were conducted under sink conditions in accordance with the pharmacopoeia requirements, while only a few publications have reported the occurrence of typical "spring-and parachute" release behaviour under non-sink conditions [216, 226].

During the dissolution of crystalline drug particles, the drug molecules are gradually dissolved into the dissolution medium until they reach an equilibrium solubility $C_{\text {eq }}$ (I in Figure 7). When fiber-based ASDs composed of an API and a water soluble polymer contact the dissolution media, they should "spring" the amorphous drug molecules into the dissolution medium to generate a highly supersaturated drug solution, just as for ASDs prepared using more traditional pharmaceutical techniques [284]. However, the supersaturated solution is thermodynamically unstable: the amount of drug in solution will gradually return to a lower value, often greater than $C_{\text {eq }}$ because the polymer carriers can have solubilising effects ("II" in Figure 7). Electrospun ternary or even quaternary ASDs composed of an API and multiple excipients should provide a better "parachute" than the single polymer in binary systems, thus preventing the rapid decline of drug concentration and extending the supersaturation region (line III in Figure 7).

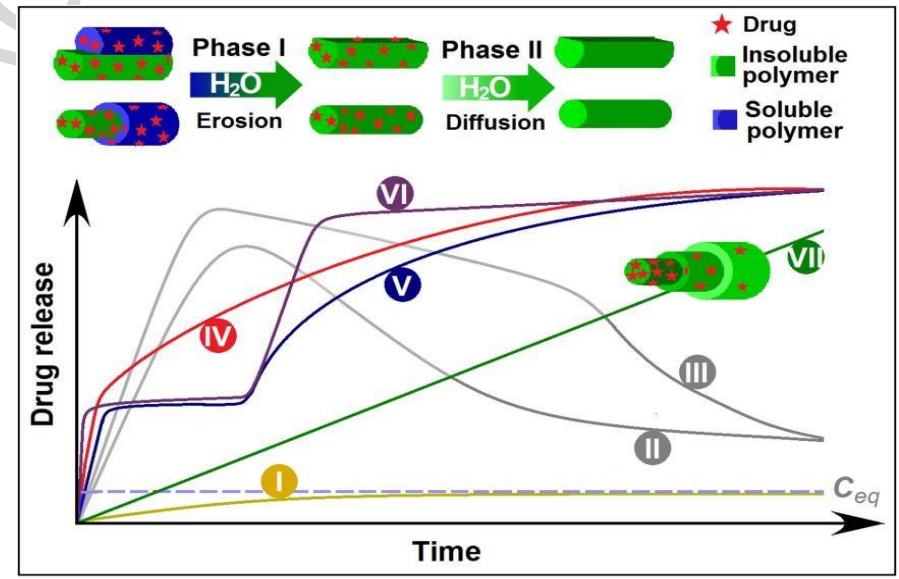


Fig. 7. Drug release profiles from different types of electropsun ASDs.

The introduction of insoluble polymers in creating ASDs is a milestone in this field. Because of the limited volume of body fluids, the drug released from an ASD-based dosage form is often under non-sink conditions, leading to the possibility of released drug molecules precipitating in the gastrointestinal tract. Thus the fast dissolution of ASDs to form supersaturated solutions can lead to suboptimal in vivo pharmacokinetic performance [285, 286]. The use of insoluble polymers as matrices to create fourth-generation ASDs not only can provide a sustained release profile, but also the gradual and extended formation of supersaturated solutions [287, 288].

Using cast films from the solvent evaporation method, Sun and Lee recently compared the effects of polymers with different solubility as drug carriers in preparing ASDs of indomethacin, including water soluble polymers (e.g. Soluplus and PVP), insoluble drug carriers (ethylcellulose and Eudragit RLPO), and $\mathrm{pH}$-sensitive polymers (e.g. hydroxypropyl methylcellulose acetate succinate, Eudragit E100 and L100) [289]. They discovered that the dissolution of the amorphous drug from soluble carriers is governed by a dissolution controlled mechanism leading to an initial surge of supersaturation followed by a sharp decline in drug concentration. In contrast, dissolution from ASDs made with insoluble polymers is regulated by a diffusioncontrolled mechanism, by which supersaturation is built up gradually and sustained over an extended period of time without any apparent decline. This is also demonstrated in other publications [290-292]. These results suggest that different release mechanisms can be exploited when designing new ASDs for poorly watersoluble APIs, potentially leading to multiple-phase release profiles and improved bioavailability. Double-fluid and multiple-fluid electrospinning processes are able to fabricate fifth-generation ASDs consisting of both soluble and insoluble drug carriers with controllable spatial distributions.

Biphasic release (Table 3) can offer effective, safe and convenient delivery of many APIs including non-steroidal anti-inflammatory drugs, antihypertensive, antihistaminic and anti-allergic agents [127,204]. Many different types of dosage forms providing biphasic release have been reported in the literature [89,201,293295]. Biphasic release is different from the simple occurrence of an uncontrolled initial burst of release; the latter is a negative phenomenon frequently seen with sustained release profiles [239], particularly in monolithic electrospun medicated nanofibers composed of a drug encapsulated in a water insoluble polymer [156]. Biphasic release is a controlled process, giving an initial rapid release of a fraction of the dose shortly after administration (a "loading dose" for swift relief of symptoms) and subsequently a sustained release of the remaining drug content allowing clinicians to optimize therapy and avoid repeated administration, hence improving patient 
convenience. This can ensure a gradual accumulation of API in the GI fluids, leading to higher bioavailability. The amount of drug released initially [296], the release site (e.g. the colon) $[127,205]$, and the release rate in the second phase can all be tuned [207]. Multi-layered nanofiber mats [297] and nanoparticle-loaded nanofibers [298] can also be employed to give biphasic release, and this area of ASD development holds much promise for future developments. 
Table 3 Biphasic release profiles provided by electrospun fibers.

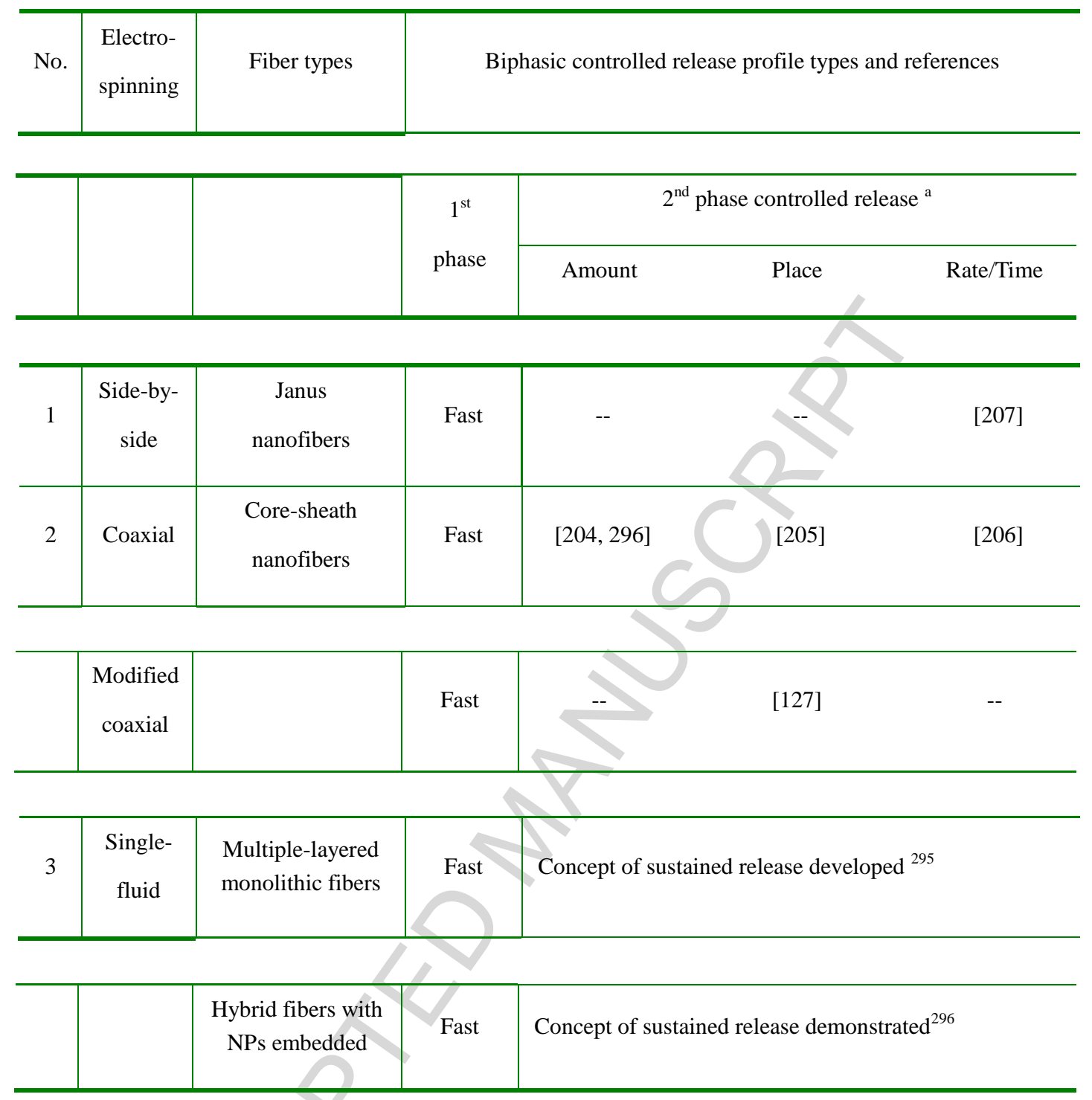

${ }^{a}$ All second phases are sustained release except when stated otherwise.

Lines IV, V and VI in Figure 7 reflect typical biphasic release profiles which can be achieved from core-shell fiber ASDs. Line IV can be delivered from ASDs consisting of a soluble shell and an insoluble core, both loaded with drug. For example, coreshell nanofibers with PVP as the shell and EC as the core can give this release profile, through a combination of dissolution and diffusion drug release mechanisms [204]. Profile V is similar to IV in that both can be considered to be a combination of second and fourth-generation ASDs. However, for $\mathrm{V}$ the carrier in the core is $\mathrm{pH}$-sensitive and can target the drug at (for instance) the colonic region, as demonstrated by reports of PVP / Eudragit L100 shell / core fibers [205, 277]. Profile VI provides two pulsatile bursts of release, one rapid at the initial stages of the dissolution test in acid conditions when the shell dissolves, and later when the fibers are transferred into a 
neutral medium, a second "spring" is delivered by the core [206].

Although core/shell systems have been most widely explored, all these release profiles can be duplicated using the Janus structure. Here, one side of the fibers provides the first rapid phase of release and the other a sustained, time-lagged or pulsatile second phase (as depicted in Figure 7). Recently, a new type of Janus fiberbased ASDs were fabricated using a Teflon-coated spinneret. The Janus fibers not only can provide biphasic release of incorporated drug, but also can be tuned to manipulate the drug release rates or time periods to follow biorhythms and ensure maximum absorbance [207]. Another recent development in structured ASDs reports tri-layer nanofibers aiming to provide linear release profiles (profile VII in Figure 7); a gradient distribution of drug was used to compensate the decrease of surface area moving from the outside to the inside of the fibers, and the gradually increasing diffusion distance [299].

\section{Conclusions and Perspectives}

The development of dosage forms of poorly water-soluble APIs remains one of the biggest challenges in the field of pharmaceutics. Different generations of amorphous solid dispersions (ASDs) have been developed and shown to be potent in improving the solubility and dissolution rates of poorly water-soluble APIs. Among these, a recent family of materials to emerge - electrospun nanofiber-based ASDs - have been found to be excellent candidates for resolving the challenges associated with such active ingredients. Electrospun ASDs have a series of advantages over their counterparts prepared by other routes in terms of both their fabrication and their functional properties. Electrospun ASDs can be generated from liquid systems such as solutions, melts, suspension, emulsions and melt-solvents with various types of APIs including Class II/IV chemical entities or functional bio-macromolecules. The electrospinning process has developed very rapidly, and as a result monolithic second, third, and fourth-generation ASDs have been reported. These are potent in enhancing the dissolution rate of drugs, improving the permeation of bio-membranes, and elevating bioavailability. However, the most attractive feature of the electrospinning approach is the creation of fifth-generation ASDs with structured nanoscale architectures, using double or multiple fluid processing. This can provide programmed drug release profiles in addition to the enhancement of dissolution.

Electrospun ASDs typically form as continuous 3D web structures which can improve both the physical stability and the dissolution rate/solubility of loaded APIs. The selection of the polymer carrier has a profound influence on the formation of the fibers and their stability. The presence of secondary interactions such as H-bonding between the drug and polymer is desireable to ensure the formation of homogeneous 
structures with high drug loadings and maintaining long-term stability of the amorphous form of the API [300]. To characterize the properties and evaluate stability and functional performance of electrospun ASDs, methods such as SEM, TEM, XRD, DSC, FTIR and Raman spectroscopy can be used with the well-known pharmacopoeia testing protocols, in addition to a number of novel approaches. A series of release profiles can be achieved including immediate release, pulsatile release, sustained release, and linear release. In particular, a number of biphasic release profiles have been reported using core-shell and Janus nanofibers.

In the future, increasing numbers of ever more complex electrospinning processes can be envisaged for creating increasingly exquisite ASDs. Further developments are likely to combine electrospinning with other more traditional pharmaceutical techniques. Additionally, more and more attention has been paid to the production of electrospun nanofibers for commercial development, with many exciting developments in scale-up technologies which should lead in the coming years to the preparation of electrospun ASDs on the industrial scale and products emerging on the market. Clinical investigations of the fiber-based ASDs are also expected to accelerate in the coming years [301-303]. There remain however a number of challenges in In translating electrospun nanofibers from the research laboratory to the clinic. The main barriers will be the need to produce large amounts of materials in a reproducible and quality-controlled environment, and to move the products through the various stages of development towards regulatory approval. Although scale-up can be achieved, to date there is little work exploring downstream processing of the fibers into dosage forms (e.g. tablets) or to GMP-standard manufacturing.

Double and multiple-fluid electrospinning can not only create different types of nanostructures (core-shell, Janus, core-shell with Janus core, etc) but also permit the tailoring of the fiber composition and spatial distribution of the encapsulated ingredients. This will lead to increasing control of structure-property-function relationships at the nanoscale. The resultant fifth-generation SDs may permit a series of pharmaceutical challenges such as multi-phasic release and controlled release of multiple APIs from a single dosage form to be addressed, in addition to their potential in improving the dissolution of poorly water-soluble APIs.

Since electrospun nanofiber-based ASDs have a molecular distribution of poorly water-soluble APIs and other hydrophobic pharmaceutical excipients (such as phospholipids and surfactants) can be homogeneously distributed within the same matrix, the nanofibers have the potential to act as molecular self-assembly templates. Using fast-dissolving polymers and hydrophobic active ingredients, solid lipid nanoparticles and liposomes have been created in situ [16,102,230,231,304]. Such ASDs can be used as precursors for the production of self-assembled and selfemulsified drug delivery systems. They can thus act as a bridge between solid dosage 
forms with good storage stability and easy-to-administer liquid dosage forms.

\section{References}

1. Kawakami K. Modification of physicochemical characteristics of active pharmaceutical ingredients and application of supersaturatable dosage forms for improving bioavailability of poorly absorbed drugs. Adv Drug Delivery Rev 2012;64:480-95.

2. Merisko-Liversidge E, Liversidge GG. Nanosizing for oral and parenteral drug delivery: A perspective on formulating poorly-water soluble compounds using wet media milling technology. Adv Drug Delivery Rev 2011;63:427-40.

3. Kawabata Y, Wada K, Nakatani M, Yamada S, Onoue S. Formulation design for poorly water-soluble drugs based on biopharmaceutics classification system: Basic approaches and practical applications. Int J Pharm 2011;420:1-10.

4. Mitragotri S, Burke PA, Langer R. Overcoming the challenges in administering biopharmaceuticals: Formulation and delivery strategies. Nat Rev Drug Discov 2014; 13:655-72.

5. Williams HD, Trevaskis NL, Charman SA, Shanker RM, Charman WN, Pouton $\mathrm{CW}$, et al. Strategies to address low drug solubility in discovery and development. Pharmcol Rev 2013;65:315-499.

6. Kesisoglou F, Panmai S, Wu Y. Nanosizing-oral formulation development and biopharmaceutical evaluation. Adv Drug Delivery Rev 2007;59:631-44.

7. McGinity JW, Maincent P, Steinfink H. Crystallinity and dissolution rate of tolbutamide solid dispersions prepared by the melt method. J Pharm Sci 1984;73: 1441-44.

8. Sekiguchi K, Obi N. Studies on absorption of eutectic mixture. I. A comparison of the behavior of eutectic mixture of sulfathiazole and that of ordinary sulfathiazole in man. Chem Pharm Bull 1961;9:866-72.

9. Okonogi S, Oguchi T, Puttipipatkhachorn S, Yamamoto K. Improved dissolution of ofloxacin via solid dispersion. Int J Pharm 1997;156:175-80.

10. Chauhan H, Hui-Gu C, Atef E. Correlating the behavior of polymers in solution as precipitation inhibitor to its amorphous stabilization ability in solid dispersions. J Pharm Sci 2013;102:1924-35.

11. Mohan A, Gundamaraju R. In vitro and in vivo evaluation of fast-dissolving tablets containing solid dispersion of lamotrigine. Int J Pharm Investig 2015;5:5764.

12. Karavas E, Georgarakis E, Sigalas MP, Avgoustakis K, Bikiaris D. Investigation of the release mechanism of a sparingly water-soluble drug from solid dispersions in hydrophilic carriers based on physical state of drug, particle size distribution and drug-polymer interactions. Eur J Pharm Biopharm 2007;66:334-47.

13. Bley H, Fussnegger B, Bodmeier R. Characterization and stability of solid dispersions based on PEG/polymer blends. Int J Pharm 2010;390:165-73.

14. Shah S, Maddineni S, Lu J, Repka MA. Melt extrusion with poorly soluble drugs. Int J Pharm 2013;453:233-52.

15. Waard HDe, Hinrichs W, Visser M, Bologna C, Frijlink H. Unexpected differences in dissolution behavior of tablets prepared from solid dispersions with a surfactant physically mixed or incorporated. Int J Pharm 2008;349:66-73. 
16. Wang Y, Wang C, Zhao J, Ding Y, Li L. A cost-effective method to prepare curcumin nanosuspensions with enhanced oral bioavailability. J Colloid Interf Sci 2017;485:91-98.

17. Le-Ngoc Vo C, Park C, Lee BJ. Current trends and future perspectives of solid dispersions containing poorly water-soluble drugs. Eur J Pharm Biophar 2013;85, 799-813.

18. Tran PHL, Tran TTD, Park JB, Lee BJ. Controlled release systems containing solid dispersions: Strategies and mechanisms. Pharm Res 2011;28:2353-78.

19. Zahedi P, Lee PI. Solid molecular dispersions of poorly water-soluble drugs in poly (2-hydroxyethyl methacrylate) hydrogels. Eur J Pharm Biopharm 2007;65: 320-28.

20. Ngoc-Gia NT, Ha-Lien TPhuong, Toi VV, Wei D, Thao TDT. Development of a sustained release solid dispersion using swellable polymer by melting method. Pharm Res 2016;33:102-9.

21. Ozeki T, Yuasa H, Kanaya Y. Controlled release from solid dispersion composed of poly (ethylene oxide)-Carbopol interpolymer complex with various crosslinking degrees of Carbopol®. J Control Release 2000;63:287-95.

22. Hughes GA. Nanostructure-mediated drug delivery. Nanomed Nanotechnol Biol Med 2005;1:22-30.

23. Goldberg M, Langer R, Jia X. Nanostructured materials for applications in drug delivery and tissue engineering. J Biomater Sci Polym Ed 2007;18:241-68.

24. Venkataraman S, Hedrick JL, Ong ZY, Yang C, Rachel Ee PL, Hammond PT, et al. The effects of polymeric nanostructure shape on drug delivery. Adv Drug Delivery Rev 2011;63:1228-46.

25. Kim J, Piao Y, Hyeon T. Multifunctional nanostructured materials for multimodal imaging, and simultaneous imaging therapy. Chem Soc Rev 2009;38: 372-90.

26. Yang P, Gai S, Lin J. Functionalized mesoporous silica materials for controlled drug delivery. Chem Soc Rev 2012;41:3679-98.

27. Wei H, Cheng SX, Zhang XZ, Zhou EX. Thermo-sensitive polymeric micelles based on poly(N-isopropylacrylamide) as drug carriers. Prog Polym Sci 2009;34(9):893-910.

28. Peng S, Jin G, Li L, Li K, Srinivasan M, Ramakrishna S, et al. Multi-functional electrospun nanofibres for advances in tissue regeneration, energy conversion \& storage, and water treatment. Chem Soc Rev 2016;45:1225-41.

29. Starr JD, Budi MAK, Andrew JS. Processing-property relationships in electrospun Janus-type biphasic ceramic nanofibers. J Am Ceram Soc 2015;98:12-9.

30. Labbaf S, Deb S, Camma G, Stride E, Edirisinghe M. Preparation of multicomponent drug delivery nanoparticles using a triple-needle electrohydrodynamic device. J Colloid Interf Sci 2013;409:245-54.

31. Jiang S, Duan G, Zussman E, Greiner A, Agarwal S. Highly flexible and tough concentric tri-axial polystyrene fibers. ACS Appl Mater Interfaces 2014;6:591823.

32. Tong R, Tang L, Ma L, Tu C, Baumgartner R, Cheng J. Smart chemistry in polymeric nanomedicine. Chem Soc Rev 2014;43:6982-7012.

33. Hu J, Zhou S, Sun Y, Fang X, Wu L. Fabrication, properties and applications of Janus particles. Chem Soc Rev 2012;41:4356-78. 
34. Labbaf S, Ghanbar H, Stride E, Edirisinghe M. Preparation of multilayered polymeric structures using a novel four-needle coaxial electrohydrodynamic device. Macromol Rapid Commun 2014;35:618-23.

35. Liu W, Ni C, Chase DB, Rabolt JF. Preparation of multilayer biodegradable nanofibers by triaxial electrospinning. ACS Macro Lett 2013;2:466-8.

36. Starr JD, Andrew JS. A Route to Synthesize multifunctional tri-phase nanofibers. J Mater Chem C 2013;1:2529-33.

37. Si Y, Chen M, Wu L. Syntheses and biomedical applications of hollow micronano-spheres with large-through-holes. Chem Soc Rev 2016;45:690-714.

38. Gawande MB, Goswami A, Asefa T, Guo H, Biradar AV, Peng DL, et al. Coreshell nanoparticles: synthesis and applications in catalysis and electrocatalysis. Chem Soc Rev 2015;44:7540-90.

39. Qi J, Lai X, Wang J, Tang H, Ren H, Yang Y, et al. Multi-shelled hollow micronanostructures. Chem Soc Rev 2015;44:6749-73.

40. Chaudhuri RG, Paria S. Core shell nanoparticles: classes, properties, synthesis mechanisms, characterization, and applications. Chem Rev 2012;112:2373-433.

41. Liu K, Xu Z, Yin M. Perylenediimide-cored dendrimers and their bioimaging and gene delivery applications. Prog Polym. Sci 2015;46:25-54.

42. Li H, John JV, Byeon SJ, Heo MS, Sung JH, Kim KH, et al. Controlled accommodation of metal nanostructures within the matrices of polymer architectures through solution-based synthetic strategies. Prog Polym Sci 2014;39:1878-907.

43. Dosio F, Arpicco S, Stella B, Fattal E. Hyaluronic acid for anticancer and nucleic acid delivery. Adv Drug Delivery Rev 2016;97:204-236.

44. Kanaujia P, Poovizhi P, Ng WK, Tan RBH. Amorphous formulations for dissolution and bioavailability enhancement of poorly soluble APIs. Powder Technol 2015;285:2-15.

45. Vasconcelos T, Sarmento B, Costa P. Solid dispersions as strategy to improve oral bioavailability of poor water soluble drugs. Drug Discov Today 2007; 12:1068-75.

46. Chokshi RJ, Zia H, Sandhu HK, Shah NH, Malick WA. Improving the dissolution rate of poorly water soluble drug by solid dispersion and solid solution-pros and cons. Drug delivery 2007;14:33-45.

47. Sethia S, Squillante E. Solid dispersions: Revival with greater possibilities and applications in oral drug delivery. Crit Rev Ther Drug Carrier System 2003;20:215-47.

48. Bikiaris DN. Solid dispersions, Part II: new strategies in manufacturing methods for dissolution rate enhancement of poorly water-soluble drugs. Exp Opin Drug Del 2011;8:1663-80.

49. Bikiaris DN. Solid dispersions, Part I: recent evolutions and future opportunities in manufacturing methods for dissolution rate enhancement of poorly watersoluble drugs. Exp Opin Drug Del 2011;8:1501-19.

50. Pasquali I, Bettini R, Giordano F. Supercritical fluid technologies: an innovative approach for manipulating the solid-state of pharmaceuticals. Adv Drug Delivery Rev 2008;60:399-410.

51. Qian F, Huang J, Hussain MA. Drug-polymer solubility and miscibility: Stability consideration and practical challenges in amorphous solid dispersion development. J Pharm Sci 2010;99:2941-47. 
52. Yasuji T, Takeuchi H, Kawashima Y. Particle design of poorly water-soluble drug substances using supercritical fluid technologies. Adv Drug Delivery Rev 2008;60:388-98.

53. Singh A, Van den Mooter G. Spray drying formulation of amorphous solid dispersions. Adv Drug Delivery Rev 2016;100:27-50.

54. Paola Aline Amarante B, Mariha P, Carlos Eduardo Maduro DC, Pezzini BR, Stulzer HK. Sodium alginate as a potential carrier in solid dispersion formulations to enhance dissolution rate and apparent water solubility of BCS II drugs. Carbohydr Polym 2016;137:350-9.

55. Paudel A, Worku ZA, Meeus J, Guns S, Van den Mooter G. Manufacturing of solid dispersions of poorly water soluble drugs by spraying: formulation and process considerations. Int J Pharm 2013;453:253-84.

56. Kolašinac N, Kachrimanis K, Homšek I, Grujić B, Durić Z, Ibrić S. Solubility enhancement of desloratadine by solid dispersion in poloxamers. Int. J. Pharm 2012;436:161-70.

57. Fini A, Rodriguez L, Cavallari C, Albertini B, Passerini N. Ultrasound compacted and spray-congealed indomethacin polyethyleneglycol systems. Int $\mathrm{J}$ Pharm 2002;247:11-22.

58. Leeke GA, Lu T, Bridson RH, Seville JPK. Application of nano-particle coatings to carrier particles using an integrated fluidized bed supercritical fluid precipitation process. J Supercrit Fluids 2014;91:7-14.

59. Patterson JE, James MB, Forster AH, Lancaster RW, Butler JM, Rades T. Preparation of glass solutions of three poorly water soluble drugs by spray drying, melt extrusion and ball milling. Int J Pharm 2007;336:22-34.

60. Liu X, Lu M, Guo Z, Huang L, Feng X, Wu C. Improving the chemical stability of amorphous solid dispersion with cocrystal technique by hot melt extrusion. Pharm Res 2012;29:806-17.

61. Lakshman JP, Cao Y, Kowalski J, Serajuddin ATM. Application of melt extrusion in the development of a physically and chemically stable high-energy amorphous solid dispersion of a poorly water-soluble drug. Mol Pharm 2008;5:994-1002.

62. Maxx C, Rajesh D. Enhanced physical stability of amorphous drug formation via dry polymer coating. J Pharm Sci 2015;104:2076-84.

63. Yu DG, Branford-White C, White K, Li XL, Zhu LM. Dissolution improvement of electrospun nanofiber-based solid dispersions for acetaminophen. AAPS Pharm Sci Tech 2010;11:809-17.

64. Verreck G, Chun I, Peeters J, Rosenblatt J, Brewster ME. Preparation and characterization of nanofibers containing amorphous drug dispersion generated by electrostatic spinning. Pharm Res 2003;20:810-17.

65. Démuth B, Farkas A, Pataki H, Balogh A, Szabó B, Borbás E, et al. Detailed stability investigation of amorphous solid dispersions prepared by single-needle and high speed electrospinning. Int J Pharm 2016;498:234-44.

66. Passerini N, Albertini B, Perissutti B, Rodriguez L. Evaluation of melt granulation and ultrasonic spray congealing as techniques to enhance the dissolution of praziquantel. Int J Pharm, 2006;318:92-102.

67. Noyes A, Whitney WR. The rate of dissolution of solid substances in their own solutions. J Am Chem Soc 1897;19:930-4.

68. Merisko-Liversidge EM, Liversidge GG. Drug nanoparticles: Formulating poorly water-soluble compounds. Toxicol Pathol 2008;36:43-8. 
69. Rasenack N, Muller BW. Dissolution rate enhancement by in situ micronization of poorly water-soluble drugs. Pharm Res 2002;19:1894-900.

70. Sarah LT, Tejal AD. Microfabricated drug delivery systems: from particles to pores. Adv Drug Delivery Rev 2003;55:315-28.

71. Lu Y, Chen SC. Micro and nano-fabrication of biodegradable polymers for drug delivery. Adv Drug Delivery Rev 2004;56:1621-33.

72. Merisko-Liversidge E, Liversidge GG, Cooper ER. Nanosizing: A formulation approach for poorly-water-soluble compounds. Eur J Pharm Sci 2003;18: 113-20.

73. Farokhzad OC. Nanotechnology for drug delivery: the perfect partnership. Expert Opin. Drug Del 2008;5:927-9.

74. Khalf A, Madihally SV. Recent advances in multiaxial electrospinning for drug delivery. Eur J Pharm Biopharm 2017;112:1-17.

75. Lee $\mathrm{J}, \mathrm{Yu} \mathrm{C}$. Critical freezing rate in freeze drying nanocrystal dispersions. J Control Release 2006;111:185-192.

76. Jia L, Shen J, Li Z, Zhang D, Zhang Q, Liu G, et al. In vitro and in vivo evaluation of paclitaxel-loaded mesoporous silica nanoparticles with three pore sizes. Int J Pharm 2013;445:12-9.

77. Doadrio AL, Sousa EM, Doadrio JC, Perez Pariente J, Izquierdo-Barba I, ValletRegi M. Mesoporous SBA-15 HPLC evaluation for controlled gentamicin drug delivery. J Control Release 2004;97:125-32.

78. Prokopowicz M, Przyjazny A. Synthesis of sol-gel mesoporous silica materials providing a slow release of doxorubicin. J Microencapsul 2007;24: 682-701.

79. Zhang Y, Zhi Z, Jiang T, Zhang J, Wang Z, Wang S. Spherical mesoporous silica nanoparticles for loading and release of the poorly water-soluble drug telmisartan. J Control Release 2010;145:257-63.

80. Ambrogi V, Perioli L, Pagano C, Marmottini F, Ricci M, Sagnella A, et al. Use of SBA-15 for furosemide oral delivery enhancement. Eur J Pharm Sci 2012;46: 43-8.

81. Kesisoglous F, Panmai S, Wu Y. Nanosizing - oral formulation development and biopharmaceutical evaluation. Adv Drug Delivery Rev 2007;59:631-44.

82. Hubbell JA, Chikoti A. Nanomaterials for drug delivery. Science 2012;337: 3035.

83. Zhang $\mathrm{K}$, Yu H, Luo Q, Yang S, Lin X, Zhang Y, et al. Increased dissolution and oral absorption of itraconazole Soluplus extrudate compared with itraconazole nanosuspension. Eur J Pharm Biopharm 2013;85:1285-92.

84. Fule R, Meer T, Sav A, Amin P. Solubility and dissolution rate enhancement of lumefantrine using hot melt extrusion technology with physicochemical characterization. J Pharm Investig 2013;43:305-21.

85. Salata OV. Tools of nanotechnology: Electrospray. Curr Nanosci 2005;1: 25-33.

86. Chakraborty S, Liao IC, Adler A, Leong KW. Electrohydrodynamics: A facile technique to fabricate drug delivery systems. Adv Drug Delivery Rev 2009;61: 1043-54.

87. Ding L, Lee T, Wang CH. Fabrication of monodispersed taxol-loaded particles using electrohydrodynamic atomization. J Control Release 2005;102:395-413.

88. George MC, Braun PV. Multicompartmental materials by electrohydrodynamic co-jetting. Angew Chem Int Ed 2009;48:8606-09.

89. Yu DG, Zhu LM, Branford-White C, Yang XL. Three-dimensional printing in pharmaceutics - promises and problems. J Pharm Sci 2008;97:3666-90.

90. Yu DG, Yang X, Huang W, Xu H, Wang Y. Material gradients drug delivery systems fabricated by three-dimensional printing. J Pharm Sci 2007;96:2446-56. 
91. Góra A, Sahay R, Thavasi V, Ramakrishna S. Melt-electrospun fibers for advances in biomedical engineering, clean energy, filtration, and separation. Polym Rev 2011;51:265-87.

92. Balogh A, Farkas B, Farago K, Farkas A, Wagner I, Assche IV, et al. Melt-blown and electrospun drug-loaded polymer fiber mats for dissolution enhancement: A comparative study. J Pharm Sci 2015;104:1767-76.

93. Nagy ZK, Balogh A, Drávavölgyi G, Ferguson J, Pataki H, Vajna B, et al. Solvent-free melt electrospinning for preparation of fast dissolving drug delivery system and comparison with solvent-based electrospun and melt extruded systems. J Pharm Sci 2013;102:508-17.

94. Sarkar K, Gomez C, Zambrano S, Ramirez M, Hoyos ED, Vasquez H, et al. Electrospinning to Forcespinning ${ }^{\mathrm{TM}}$. Mater Today 2010;13:12-4.

95. Chang WM, Wang CC, Chen CY. The combination of electrospinning and forcespinning: Effects on a viscoelastic jet and a single nanofiber. J Chem Eng 2014; 244:540-51.

96. Lin YN, Clark DM, Yu X, Zhong ZX, Liu KY, Reneker HD. Mechanical properties of polymer nanofibers revealed by interaction with streams of air. Polymer 2012;53:782-90.

97. Zhmayev E, Cho D, Joo YL. Nanofibers from gas-assisted polymer melt electrospinning. Polymer 2010;51:4140-4.

98. Givens SR, Gardner KH, Rabolt JF, Chase DB. High-temperature electrospinning of polyethylene microfibers from solution. Macromolecules 2007;40: 608-10.

99. Wang C, Chien HS, Hsu CH, Wang YC, Wang CT, Lu HA. Electrospinning of polyacrylonitrile solutions at elevated temperatures. Macromolecules 2007;40: 7973-83.

100.Yu DG, Gao LD, White K, Brandford-White C, Lu WY, Zhu LM. Multicomponent amorphous nanofibers electrospun from hot aqueous solutions of a poorly soluble drug. Pharm Res 2010;27:2466-77.

101.Yu DG, Branford-White C, Wang X, Li X, Qian W, Li Y. Solid dispersion prepared by electrospraying. J Biomed Sci Eng 2011;4:741-9.

102.Yu DG, Williams GR, Yang JH, Wang X, Yang JM, Li XY. Solid lipid nanoparticles self-assembled from electrosprayed polymer-based micoparticles. J Mater Chem 2011;21:15957-61.

103.Li D, Xia Y. Electrospinning of nanofibers: Reinventing the wheel. Adv Mater 2004;16:1151-70.

104.Rutledge GC, Fridrikh SV. Formation of fibers by electrospinning. Adv Drug Delivery Rev 2007;59:1384-91.

105.Sridhar R, Lakshminarayanan R, Madhaiyan K, Barathi VA, Limh KHC, Ramakrishna S. Electrosprayed nanoparticles and electrospun nanofibers based on natural materials: Applications in tissue regeneration, drug delivery and pharmaceuticals. Chem Soc Rev 2015;44:790-814.

106.Williams GR, Chatterton NP, Nazir T, Yu DG, Zhu LM, Branford-White C. Electrospun nanofibers in drug delivery: recent developments \& perspectives. Ther Delivery 2012;3:515-33.

107. Lee KJ, Yoon J, Lahann J. Recent advances with anisotropic particles. Curr Opin Colloid Interf Sci 2011;16:195-202.

108.Nagy ZK, Balogh A, Démuth B, Pataki H, Vigh T, Szabó B, ey al. High speed electrospinning for scaled-up production of amorphous solid dispersion of itraconazole. Int. J. Pharm 2015;480:137-42. 
109.Molnar K, Nagy ZK. Corona-electrospinning: Needleless method for highthroughput continuous nanofiber production. Eur J Pharm 2016;74:279-86.

110.Zhang H, Niu Q, Wang N, Nie J, Ma G. Thermo-sensitive drug controlled release PLA core PNIPAM shell fibers fabricated using a combination of electrospinning and UV photo-polymerization. Eur J Pharm 2015;71:440-50.

111.Agarwal S, Greiner A, Wendorff JH. Functional materials by electrospinning of polymers. Prog Polym Sci 2013;38:963-91.

112.Zhang CL, Yu SH. Nanoparticles meet electrospinning: Recent advanced and future prospects. Chem Soc Rev 2014;43:4423-48.

113.Liu W, Thomopoulos S, Xia Y. Electrospun nanofibers for regenerative medicine. Adv Healthcare Mater 2012;1:10-25.

114.Zhao Y, Cao X, Jiang L. Bio-mimic multichannel microtubes by a facile method. J Am Chem Soc 2007;129:764-5.

115.Yu DG, Branford-White C, Bligh SWA, White K, Chatterton NP, Zhu LM. Improving polymer nanofiber quality using a modified co-axial electrospinning process. Macromol Rapid Commun 2011;32:744-50.

116.Zheng J, Yan X, Li MM, Yu GF, Zhang HD, Pisula W, He XX. Electrospun aligned fibrous arrays and twisted ropes: Fabrication, mechanical and electrical properties, and application in strain sensors. Nanoscale Res Lett 2015;10:475.

117.Long YZ, Yu M, Sun B, Gu CZ, Fan ZY. Recent advances in large-scale assembly of semiconducting inorganic nanowires and nanofibers for electronics, sensors and photovoltaics. Chem Soc Rev 2012;41:4560-80.

118.Balogh A, Cselkó R, Démuth B, Verreck G, Mensch J, Marosi G, et al. Alternating current electrospinning for preparation of fibrous drug delivery systems. Int J Pharm 2015;495:75-80.

119.Li W, Yu DG, Chen K, Wang G, Williams GR. Smooth preparation of ibuprofen zein microcomposites using an epoxy-coated electrospraying head. Mater Lett 2013;93:125-8.

120.Liu ZP, Cui L, Yu DG, Zhao ZX, Chen L. Electrosprayed core-shell solid dispersions of acyclovir fabricated using an epoxy-coated concentric spray head. Int J Nanomed 2014;9:1967-77.

121.Wang X, Li XY, Li Y, Zou H, Yu DG, Cai JS. Electrospun acetaminophenloaded cellulose acetate nanofibers fabricated using an epoxy-coated spinneret. E-Polymers 2015;15:311-5.

122.Li C, Yu DG, Williams GR, Wang ZH. Fast-dissolving core-shell composite microparticles of quercetin fabricated using a coaxial electrospray process. PLOS One 2014;9:e92106.

123.Li C, Wang ZH, Yu DG. Higher quality quercetin sustained release ethyl cellulose nanofibers fabricated using a spinneret with a Teflon nozzle. Colloids Surf B 2014;114:404-9.

124.Li C, Wang ZH, Yu DG, Williams GR. Tunable biphasic drug release from ethyl cellulose nanofibers fabricated using a modified coaxial electrospinning process. Nanoscale Res Lett 2014;9:258.

125.Xiang Q, Ma YM, Yu DG, Jin M, Williams GR. Electrospinning using a Tefloncoated spinneret. Appl Surf Sci 2013;284:889-93.

126. Yarin AL, Koombhongse S, Reneker DH. Taylor cone and jetting from liquid droplets in electrospinning of nanofibers. J Appl Phys 2001;90:4836.

127.Yu DG, Williams GR, Wang X, Liu XK, Li HL, Bligh SWA. Dual drug release nanocomposites prepared using a combination of electrospraying and electrospinning. RSC Adv 2013;3:4652-8. 
128.Kriegel C, Kit KM, McClements DJ, Weiss J. Influence of surfactant type and concentration on electrospinning of chitosan-poly(ethylene oxide) blend nanofibers. Food Biophys 2009;4:213-28.

129. Yang JM, Yu DG. Co-axial electrospinning with sodium thiocyanate solution for preparing polyacrylonitrile nanofibers. J Polym Res 2012;19:9789 (7pp).

130.Yu DG, Williams GR, Gao LD, Annie Bligh SW, Yang JH, Wang X. Coaxial electrospinning with sodium dodecylbenzene sulfonate solution for high quality polyacrylonitrile nanofibers. Colloids Surf A 2012;396:161-8.

131.Yu DG, Lu P, White CB, Yang JH, Wang X. Polyacrylonitrile nanofibers prepared using co-axial electrospinning with $\mathrm{LiCl}$ solution as sheath fluid. Nanotechnology 20111;22:435301.

132.Yu DG, White K, Yang JH, Wang X, Qian W, Li Y. PVP nanofibers prepared using co-axial electrospinning with salt solution as sheath fluid. Mater Lett 2012;67:78-80.

133.Yu DG, Chatterton NP, Yang JH, Wang X, Liao YZ. Coaxial electrospinning with triton X-100 solutions as sheath fluids for preparing PAN nanofibers. Macromol Mater Eng 2012;297:395-401.

134.Ziabicki A. Fundamentals of fiber formation, John Wiley and Sons, London, 1976, ISBN 0-471-98220-2.

135.Bhat PP, Appathurai S, Harris MT, Pasquali M, McKinley GH, Basaran OA. Formation of beads-on-a-string structures during break-up of viscoelastic filaments. Nat Phys 2010;6:625-31.

136.Fong H, Chun I, Reneker DH. Beaded nanofibers formed during electrospinning. Polymer 1999;40:4585-92.

137.Yang JH, Yang GZ, Yu DG, Wang X, Zhao B, Zhang L, et al. Carbon foams from polyacrylonitrile-borneol films prepared using coaxial electrohydrodynamic atomization. Carbon 2013;53:231-6.

138.Enayati M, Ahmad Z, Stride E, Edirisinghe M. One-step electrohydrodynamic production of drug-loaded micro- and nanoparticles. J R Soc Interface 2010;7: 667-75.

139.Ramakrishna S, Fujihara K, Teo WE, Yong T, Ma Z, Ramaseshan R. Electrospun nanofibers: solving global issues. Mater Today 2006;9:40-50.

140.Doshi J, Reneker DH. Electrospinning process and applications of electrospun fibers. J Electrostat 1995;35:151-60.

141.Sebs I, Szabó P, Kállai-Szabó B, Zelkó R. Incorporating small molecules or biologics into nanofibers for optimized drug release: A review. Int $\mathrm{J}$ Pharm 2015;494: 516-30.

142.Illangakoon UE, Nazir T, Williams GR, Chatterton NP. Mebeverine-loaded electrospun nanofibers: physicochemical characterization and dissolution studies. J Pharm Sci 2014;103:283-92.

143.Li X, Kanjwal MA, Lin L, Chronakis IS. Electrospun polyvinyl-alcohol nanofibers as oral fast-dissolving delivery system of caffeine and riboflavin. Colloids Surf B 2013;103:182-8.

144. Ohara T, Kitamura S, Kitagawa T, Terada K. Dissolution mechanism of poorly water-soluble drug from extended release solid dispersion system with ethylcellulose and hydroxypropylmethylcellulose. Int J Pharm 2005;302:95-102.

145.Lopez FL, Shearman GC, Gaisford S, Williams GR. Amorphous formulations of indomethacin and griseofulvin prepared by electrospinning. Mol Pharm 2014;11:4327-38. 
146.Wu YH, Yu DG, Li XY, Diao AH, Illangakoon UE, Williams GR. Fastdissolving sweet sedative nanofiber membranes. J Mater Sci 2015;50:3604-13.

147. Yu DG, Branford-White C, Shen XX, Zhang XF, Zhu LM. Solid dispersions of ketoprofen in drug-loaded electrospun nanofibers. J Dispersion Sci Technol 2010;31:902-8.

148.Balogh A, Drávavölgyi G, Faragó K, Farkas A, Vigh T, Sóti PL, et al. Plasticized drug-loaded melt electrospun polymer mats: characterization, thermal degradation, and release kinetics. J Pharm Sci 2014;103:1278-87.

149.Yu DG, Yang JM, Branford-White C, Lu P, Zhang L, Zhu LM. Third generation solid dispersions of ferulic acid in electrospun composite nanofibers. Int $\mathbf{J}$ Pharm 2010;400:158-64.

150.Chen G, Xu Y, Yu DG, Zhang DF, Chatterton NP, White KN. Structure-tunable Janus fibers fabricated using spinnerets with varying port angles. Chem Commun 2015;51:4623-6.

151.Walther A, Müller AHE. Janus particles: synthesis, self-assembly, physical properties, and applications. Chem Rev 2013;113:5194-261.

152.Perez RA, Kim HW. Core-shell designed scaffolds for drug delivery and tissue engineering. Acta Biomater 2015;21:2-19.

153.Davoodi P, Feng F, Xu Q, Yan WC, Tong YW, Srinivasan MP, et al. Coaxial electrohydrodynamic atomization: Microparticles for drug delivery applications. J Control Release 2015;205:70-82.

154.Dzenis AY. Spinning continuous fibers for nanotechnology. Science 2004;304:1917-19.

155.Moghe AK, Gupta BS. Co-axial electrospinning for nanofiber structures: Preparation and applications. Polym Rev 2008;48:353-77.

156.Yu DG, Yu JH, Chen L, Williams GR, Wang X. Modified coaxial electrospinning for the preparation of high-quality ketoprofen-loaded cellulose acetate nanofibers. Carbohydr Polym 2012;90:1016-23.

157.Yu DG, Branford-White C, Chatterton NP, White K, Zhu LM, Shen XX, et al. Electrospinning of concentrated polymer solutions. Macromolecules 2010;43: 10743-6.

158.Yu DG, Branford-White C, White K, Chatterton NP, Zhu LM, Huang LY, et al. A modified coaxial electrospinning for preparing fibers from a high concentration polymer solution. EXPRESS Polym Lett 2011;5:732-41.

159.Li XY, Yu DG, Liao YZ, Wang X. Fast disintegrating quercetin-loaded drug delivery systems fabricated using coaxial electrospinning. Int J Mol Sci 2013;14: 21647-59.

160.Yu DG, Li XY, Chian W, Li Y, Wang X. The influence of sheath solvents on the quality of ethyl cellulose nanofibers from a modified coaxial electrospinning. Bio-Med Mater Eng 2014;24:695-701.

161.Yu DG, Liao Y, Li X, Chian W, Li Y, Wang X. Colon-targeted drug delivery core-sheath nanofibers prepared using a modified coaxial electrospinning. J Control Release 2014;172:e26.

162.Yu DG, Xu Y, Li Z, Du LP, Zhao BG, Wang X. Coaxial electrospinning with mixed solvents: From flat to round Eudragit L100 nanofibers for better colontargeted sustained drug release profiles. J Nanomater 2014;2014:967295.

163.Gupta P, Wilkes GL. Some investigations on the fiber formation by utilizing a side-by-side bicomponent electrospinning approach. Polymer 2003;44:6353-9. 
164.Starr JD, Budi MAK, Andrew JS. Processing- property relationships in electrospun Janus- type biphasic ceramic nanofibers. J Am Ceram Soc 2015;98:12.

165.Zhou X, Ma Q, Dong X, Wang J, Yu W, Liu G. Flexible Janus nanofibers: a feasible route to realize simultaneously tuned magnetism and enhanced colortunable luminescence bifunctionality. RSC Adv 2015;5:35948-57.

166.Starr JD, Andrew JS. Janus-type bi-phasic functional nanofibers. Chem Commun 2013;49:4151-3.

167.Rahmani S, Park TH, Dishman AF, Lahann J. Multimodal delivery of irinotecan from microparticles with two distinct compartments. J Control Release 2013;172:239-45.

168.Lv N, Ma Q, Dong X, Wang J, Yu W, Liu G. Flexible Janus nanofibers: Facile electrospinning construction and enhanced luminescent-electrical-magnetic trifunctionality. Chem Plus Chem 2014;79:690-7.

169.Ma QL, Yu WS, Dong XT, Wang JX, Liu GX. Janus nanobelts: fabrication, structure and enhanced magnetic-fluorescent bifunctional performance. Nanoscale 2014;6:2945-52.

170.Wang K, Liu XK, Chen XH, Yu DG, Yang YY, Liu P. Electrospun hydrophilic Janus nanocomposites for the rapid onset of therapeutic action of helicid. ACS Appl Mater Interfaces 2018; 10:2859-2867

171.Démuth B, Nagy ZK, Balogh A, Vigh T, Marosi G, Verreck G, et al. Downstream processing of polymer-based amorphous solid dispersions to generate tablet formulations. Int J Pharm 2015;486:268-86.

172. Illangakoon UE, Gill H, Shearman GC, Parhizkar M, Mahalingam S, Chatterton NP, et al. Fast dissolving paracetamol caffeine nanofibers prepared by electrospinning. I J Pharm 2014;30:369-79.

173.Wang X, Yu DG, Li XY, Bligh SWA, Williams GR. Electrospun medicated shellac nanofibers for colon-targeted drug delivery. Int J Pharm 2015;490:384-90.

174.Nagy ZK, Balogh A, Demuth B, Pataki H, Vigh T, et al.; High speed electrospinning for scaled-up production of amorphous solid dispersion of itraconazole. Int. J. Pharm 2015;480: 137-142.

175.Echenique PM. Advice to a young scientist. Science Careers 10.1126 science.caredit.a1500124, May 12, 2015.

176.Goddeeris C, Van Den Mooter G. Free flowing solid dispersions of the anti-HIV drug UC 781 with Poloxamer 407 and a maximum amount of TPGS 1000: Investigating the relationship between physicochemical characteristics and dissolution behaviour. Eur J Pharm Sci 2008;35:104-13.

177.Sun N, Wei X, Wu B, Chen J, Lu Y, Wu W. Enhanced dissolution of silymarin/polyvinylpyrrolidone solid dispersion pellets prepared by a one-step fluid-bed coating technique. Powder Technol 2007;182:72-80.

178.Yu DG, Shen XX, Branford-White C, Zhu LM, Yang XL. Novel oral fast disintegrating drug delivery devices with predefined inner structure fabricated by 3DP. J Pharm Pharmacol 2009;61:323-9.

179.Dhirendra K, Lewis S, Udupa N, Atin K. Solid dispersions: a review. Pak J Pharm Sci 2009;22:234-46.

180.Matsumoto T, Zografi G. Physical properties of solid molecular dispersions of indomethacine with PVP and PVPVA in relation to indomethacine recrystallization. Pharm Res 1999;16:1722-8.

181.Anonymity. https: en.wikipedia.org wiki Electrospinning, 07-Sep-2015. 
182.Jiang H, Wang L, Zhu K. Coaxial electrospinning for encapsulation and controlled release of fragile water-soluble bioactive agents. J Control Release 2014; 193:296-303.

183.Su Y, Su Q, Liu W, Lim M, Venugopal JR, Mo X, et al. Controlled release of bone morphogenetic protein and dexamethasone loaded in core-shell PLLACLcollagen fibers for use in bone tissue engineering. Acta Biomater 2012;8:763-71.

184.Yu DG, Zhu LM, Branford-White C, Yang JH, Wang X, Li Y, et al. Solid dispersions in the form of electrospun core-sheath nanofibers. Int $\mathbf{J}$ Nanomed 2011;6: 3271-80.

185.Tran PHL, Tran HTT, Lee BJ. Modulation of microenvironmental $\mathrm{pH}$ and crystallinity of ionizable telmisartan using alkalizers in solid dispersions for controlled release. J Control Release 2008;129:59-65.

186.Tran TTD, Tran PHL, Lim J, Park JB, Choi SK, Lee BJ. Physicochemical principles of controlled release solid dispersion containing a poorly watersoluble drug. Ther Delivery 2010;1:51-62.

187.Celebioglu A, Uyar T. Cyclodextrin nanofibers by electrospinning. Chem Commun 2010;46:6903-5.

188.McKee MG, Layman JM, Cashion MP, Long TE. Phospholipid nonwoven electrospun membranes. Science 2006;311:353-5.

189.Aytac Z, Sen HS, Durgun E, Uyar T. Sulfisoxazole cyclodextrin inclusion complex incorporated inelectrospun hydroxypropyl cellulose nanofibers as drug delivery system. Colloid Surf B 2015;128:331-8.

190.Myerson AS, Trout BL. Electrospun formulations containing crystalline active pharmaceutical ingredients. Pharm Res 2013;30:238-46.

191.Chen HM, Yu DG. An elevated temperature electrospinning process for preparing acyclovir-loaded PAN ultrafine fibers. J Mater Process Technol 2010;210: 1551-5.

192.Sun B, Long YZ, Zhang HD, Li MM, Duvail JL, Jiang XY, et al. Advances in three-dimensional nanofibrous macrostructures via electrospinning. Prog Polym Sci 2014;39:862-90.

193.Maheshwari S, Chang HC. Assembly of multi-stranded nanofiber threads through AC electrospinning. Adv Mater 2009;21:349-54.

194.Chokshi RJ, Zia H, Sandhu HK, Shah NH, Malick WA. Improving the dissolution rate of poorly water-soluble drug by solid dispersion and solid solution-pros and cons. Drug Delivery 2007;14:33-45.

195.Theron SA, Yarin AL, Zussman E, Kroll E. Multiple jets in electrospinning: Experiment and modeling. Polymer 2005;46:2889-99.

196.Thoppey NM, Bochinski JR, Clarke LI, Gorga RE. Edge electrospinning for high throughput production of quality nanofibers. Nanotechnology 2011;22:345301.

197.Sharma U, Pham Q, Marini J. Electrospinning process for fiber manufacture. US Patent 2015;9:034240.

198.Shin HU, Li Y, Paynter A, Nartetamrongsutt K, Chas GG. Vertical rod method for electrospinning polymer fibers. Polymer 2015;65:26-33.

199.Squires TM, Quake SR. Microfluidics: Fluid physics at the nanoliter scale. Rev Mod Phys 2005;77:977-1026.

200. Gandhi KJ, Deshmane SV, Biyani KR. Polymers in pharmaceutical drug delivery system: A review. Int J Pharma Sci Rev Res 2012;14:57-66.

201.Cui L, Liu ZP, Yu DG, Zhang SP, Bligh SWA, Zhao N. Electrosprayed coreshell nanopartciles of PVP and shellac for furnishing biphasic controlled release of ferulic acid. Colloid Polym Sci 2014;292:2089-96. 
202.Amidon GL, Lennernäs H, Shah VP, Crison JR. A theoretical basis for a biopharmaceutic drug classification: the correlation of in vitro drug product dissolution and in vivo bioavailability. Pharm Res 1995;12:413-20.

203.Kenawy ER, Bowlin GL, Mansfield K, Layman J, Simpson DG, Sanders EH, et al. Release of tetracycline hydrochloride from electrospun poly (ethylene-covinylacetate), poly(lactic acid), and a blend. J Control Release 2002;81:57-64.

204.Yu DG, Wang X, Li XY, Chian W, Li Y, Liao YZ. Electrospun biphasic drug release polyvinylpyrrolidone ethyl cellulose core sheath nanofibers. Acta Biomater 2013;9:5665-72.

205.Yu DG, Liu F, Cui L, Liu ZP, Wang X, Bligh SWA. Coaxial electrospinning using a concentric Teflon spinneret to prepare biphasic-release nanofibers of helicid. RSC Adv 2013;3 (39):17775-83.

206.Kaassiss AYA, Young N, Sano N, Merchant HA, Yu DG, Chatterton NP, et al. Pulsatile drug release from electrospun poly(ethylene oxide) - sodium alginate blend nanofibres. J Mater Chem B 2014;2(10):1400-07.

207.Yu DG, Yang C, Jin M, Williams GR, Zou H, Wang X, Annie Bligh SW. Medicated Janus fibers fabricated using a Teflon-coated side-by-side spinneret. Colloids Surf B 2016;138:110-6.

208.Zhao X, Zhao J, Lin ZY, Pan G, Zhu Y, Cheng Y, Cui W. Self-coated interfacial layer at organic inorganic phase for temporallycontrolling dual-drug delivery from electrospun fibers. Colloids Surf B 2015;130:1-9.

209.Perez RA, Kim HW. Core-shell designed scaffolds for drug delivery and tissue engineering. Acta biomater 2015;21:2-19.

210.Tang C, Saquing CD, Morton SW, Glatz BN, Kelly RM, Khan SA. Cross-linked polymer nanofibers for hyperthermophilic enzyme immobilization: Approaches to improve enzyme performance. ACS Appl Mat Interfaces 2014;6: 11899-906.

211.Puhl S, Li L, Meinel L, Germershaus O. Controlled protein delivery from electrospun non-wovens: Novel combination of protein crystals and a biodegradable release matrix. Mol Pharm 2014;11:2372-80.

212.Li C, Zhou L, Wang C, Liu X, Liao K. Electrospinning of a PMA-co-PAA FP biopolymer nanofiber: Enhanced capability for immobilized horseradish peroxidase and its consequence for p-nitrophenol disposal. RSC Adv 2015;5: 41994-8.

213.Wang Y, Yang J, Chen L. Convenient fabrication of electrospun prolamin protein delivery system with three-dimensional shapeability and resistance to fouling. ACS Appl Mater Interfaces 2015;7:13422-30.

214.Blasi P, D'Souza SS, Selmin F, DeLuca PP. Plasticizing effect of water on poly (lactide-co-glycolide). J Control Release 2005;108:1-9.

215. Chiou WL, Riegelman. Pharmaceutical applications for solid dispersion systems. J Pharm Sci 1971;60:1281-1302.

216.den Mooter GV. The use of amorphous solid dispersions: A formation strategy to overcome poor solubility and dissolution rate. Drug Discovery Today 2012;9:7985.

217.Paaver U, Heinämäki J, Laidmäe I, Lust A, Kozlova J, Sillaste E, et al. Electrospun nanofibers as a potential controlled-release solid dispersion system for poorly water-soluble drugs. Int J Pharm 2015;479:252-60.

218.Sridhar R, Madhaiyan K, Sundarrajan S, Gora A, Venugopal JR, Ramakrishna S. Cross-linking of protein scaffolds for therapeutic applications: PCL nanofibers delivering riboflavin for protein cross-linking. J Mater Chem B 2014;2: 1626-33. 
219.Ahmed FE, Lalia BS, Hashaikeh R. A review on electrospinning for membrane fabrication: Challenges and applications. Desalination 2015;356:15-30.

220.Liu Y, Rafailovich MH, Malal R, Cohn D, Chidambaram D. Engineering of biohybrid materials by electrospinning polymer-microbe fibers. P Natl Acad Sci USA 2009; 106:14201-06.

221.Alam MA, Al-Jenoobi FI, Al-mohizea AM. Commercially bioavailable proprietary technologies and their marketed products. Drug Discovery Today 2013;18: 936-49.

222.Ignatious F, Sun L, Lee CP, Baldoni J. Electrospun nanofibers in oral drug delivery. Pharm Res 2010;27:576-88.

223.Li XQ, Lin L, Zhu YN, Liu WW, Yu TS, Ge MQ. Preparation of ultrafine fastdissolving Cholecalciferol-Loaded Poly(vinyl pyrrolidone) fiber mats via electrospinning. Polym Compos 2013;34:282-287.

224.Yu DG, Shen XX, Branford-White C, White K, Zhu LM, Bligh SWA. Oral fastdissolving drug delivery membranes prepared from electrospun polyvinylpyrrolidone ultrafine fibers. Nanotechnology 2009;20:055104.

225.Jirsak O, Sanetrnik F, Lukas D, Kotek K, Martinova L, Chaloupek JA. method of nanofibres production from a polymer solution using electrostatic spinning and a device for carrying out the method. WO Patent 2005;024101.

226.Vrbata P, Berka P, Stránská D, Doležal P, Lázníček M. Electrospinning of diosmin from aqueous solutions for improved dissolution and oral absorption. Int J Pharm 2004;473:407-13.

227.Jin M, Yu DG, Wang X, Geraldes CFGC, Williams GR, Bligh SWA. Electrospun contrast agent-loaded fibers for colon-targeted MRI. Adv Healthc Mater 2016;5:977-85.

228.Shen XX, Yu DG, Zhu LM, Branford-White C, White K, Chatterton NP. Electrospun diclofenac sodium loaded Eudragit L100 nanofibers for colontargeted drug delivery. I J Pharm 2011;408:200-207.

229.Balogh A, Horváthová T, Fülöp Z, Loftsson T, Harasztos AH, Marosi G, et al. Electroblowing and electrospinning of fibrous diclofenac sodium-cyclodextrin complex-based reconstitution injection. J Drug Delivery Sci Technol 2015;4:2834.

230.Yu DG, Branford-White C, White K, Bligh SWA, Williams G, Zhu LM, et al. Self-assembled liposomes from amphiphilic electrospun nanofibers. Soft Matter 2011; 7:8239-47.

231.Yu DG, Zhu LM, Branford-White C, Bligh SWA, White K. Coaxial electrospinning with organic solvent for controlling the self-assembled nanoparticle size. Chem Commun 2011;47:1216-18.

232. Verreck G, Chun I, Rosenblatt J, Dijck JV, Mensch J, Noppe M, et al. Incorporation of drugs in an amorphous state into electrospun nanofibers composed of a water-insoluble, nonbiodegradable polymer. J Control Release 2003;92:349-60.

233.Im JS, Yun J, Lim YM, Kim HI, Lee YS. Fluorination of electrospun hydrogel fibers for a controlled release drug delivery system. Acta Biomater 2010;6: 1029.

234.Yan J, White K, Yu DG, Zhao XY. Sustained release multiple-component cellulose acetate nanofibers fabricated using a modified coaxial electrospinning process. J Mater Sci 2014;49:538-47. 
235.Qian W, Yu DG, Li Y, Li XY, Liao YZ, Wang X. Triple-component drug-loaded nanocomposites prepared using a modified coaxial electrospinning. J Nanomater 2013;2013:826471.

236.Sill TJ, von Recum HA. Electrospinning, Applications in drug delivery and tissue engineering. Biomaterials 2008;29:19889-2006.

237.harma R, Singh H, Joshi M, Garg T, Goyal AK, Rath G. Recent Advances in polymeric electrospun nanofibers for drug delivery. Crit Rev Ther Drug 2014;31: 187-217.

238.Yu DG, Li XY, Wang X, Chian W, Liao YZ, Li Y. Zero-order drug release cellulose acetate nanofibers prepared using coaxial electrospinning. Cellulose 2013;20: 379-89.

239.Yu DG, Chian W, Wang X, Li XY, Li Y, Liao YZ. Linear drug release membrane prepared by a modified coaxial electrospinning process. J Membr Sci 2013;428:150-156.

240. Mendes RG, Bachmatiuk A, Büchner B, Cuniberti G, Rümmeli MH. Carbon nanostructures as multi-functional drug delivery platforms. J Mater Chem B 2013;1:401-28.

241.Zhang Y, Chan HF, Leong KW. Advanced materials and processing for drug delivery, The past and the future. Adv Drug Delivery Rev 2003;65:104-20.

242.Sun J, Zhang L, Wang J, Feng Q, Liu D, Yin Q, et al. Tunable rigidity of (polymeric core)-(lipid shell) nanoparticles for regulated cellular uptake, Adv Mater 2015;27:1402-7.

243.Liang X, Li J, Joo JB, Gutierrez A, Tillekaratne A, Lee I, et al. Diffusion through the shells of yolk-shell and core-shell nanostructures in the liquid phase. Angew Chem Int Ed 2012;51:8034 -6.

244.Khalf A, Singarapu K, Madihally SV. Cellulose acetate core-shell structured electrospun fiber: fabrication and characterization. Cellulose 2015;22:1389-1400.

245.Ma Q, Wang J, Dong X, Yu W, Liu G. Flexible Janus nanoribbons array, a new strategy to achieve excellent electrically conductive anisotropy, magnetism, and photoluminescence. Adv Funct Mater 2015;25:2436-43.

246. Oh KS, Song JY, Cho SH, Lee BS, Kim SY, Kim K, et al. Paclitaxel-loaded pluronic nanoparticles formed by a temperature-induced phase transition for cancer therapy. J Control Rel 2010;148:344-50.

247.Megdi E, Eleanor S, Mohan E. Electrosprayed core-shell polymer-lipid nanoparticles for active component delivery. Nanotechnology 2013;24:465-604.

248.Huang ZM, Zhang YZ, Kotaki M, Ramakrishna S. A review on polymer nanofibers by electrospinning and their applications in nanocomposites. Compos Sci Technol 2003;63:2223-53.

249.Phuong HLT, Tran TTD, Lee SA, Nho VH, Chi SC, Lee BJ. Roles of MgO release from polyethylene glycol 6000-based solid dispersions on microenvironmental $\mathrm{pH}$, enhanced dissolution and reduced gastrointestinal damage of telmisartan. Arch Pharm Res 2011;34:747-55.

250.Heo MY, Piao ZZ, Kim TW, Cao QR, Kim A, Lee BJ. Effect of solubilizing and microemulsifying excipients in polyethylene glycol 6000 solid dispersion on enhanced dissolution and bioavailability of ketoconazole. Arch Pharm Res 2005;28: 604-11.

251.Tran PHL, Tran TTD, Piao ZZ, Van Vo T, Park JB, Lim J, et al. Physical properties and in vivo bioavailability in human volunteers of isradipine using controlled release matrix tablet containing self-emulsifying solid dispersion. Int $\mathbf{J}$ Pharm 2013;450:79-86. 
252.Russo M, Spagnuolo C, Tedesco I, Bilotto S, Russo GL. The flavonoid quercetin in disease prevention and therapy, Facts and fancies. Biochem Pharmacol 2012;83:6-15.

253.Yan J, Wu YH, Yu DG, Williams GR, Huang SM, Tao W, et al. Electrospun acid-base pair solid dispersions of quercetin. RSC Adv 2014;4:58265 -71.

254.Jalani G, Jung CW, Lee JS, Lim DW. Fabrication and characterization of anisotropic nanofiber scaffolds for advanced drug delivery systems. Int $\mathbf{J}$ Nanomed 2014;9:33-49.

255.Liao IC, Chew SY, Leong KW. Aligned core-shell nanofibers delivering bioactive proteins. Nanomedicine 2006;1:465-71.

256.Abramoff MD, Magalhães PJ, Ram, Sunanda J. Image processing with Image. Biophotonics Int 2004;11:36-42.

257.Lee JY, Bashur CA, Goldstein AS, Schmidt CE. Polypyrrole-coated electrospun PLGA nanofibers for neural tissue applications. Biomaterials 2009;30:4325-35.

258.Baird JA, Taylor LS. Fabrication and characterization properties using thermal analysis techniques.Adv. Drug Delivery Rev 2012;64:396-421.

259. Thakral S, Terban MW, Thakral NK, Suryanarayanan R. Recent advances in the characterization of amorphous pharmaceuticals by X-ray diffractometry. Adv Drug Delivery Rev 2016;100:183-93.

260.Brettmann BK, Myerson AS, Trout BL. Solid-state nuclear magnetic resonance study of the physical stability of electrospun drug and polymer solid solutions. J Pharm Sci 2012;101:2185-93.

261.Yu DG, Branford-White C, Li L, Wu XM, Zhu LM. The compatibility of acyclovir with polyacrylonitrile in the electrospun drug-loaded nanofibers. J Appl Polym Sci 2010;117:1509-15.

262. Takeuchi H, Nagira S, Tanimura S, Yamamoto H, Kawashima Y. Tabletting of solid dispersion particles consisting of indomethacin and porous silica particles. Chem Pharm Bull 2005;53:487-91.

263.Kim M, Kim JS, Park HJ, Cho WK, Cha KH, Hwang SJ. Enhanced bioavailability of sirolimus via preparation of solid dispersion nanoparticles using a supercritical antisolvent process. Int J Nanomed 2011;6:2997-3009.

264.Leuner C, Dressman J. Improving drug solubility for oral delivery using solid dispersions. Eur J Pharm Biopharm 2000;50:47-60.

265.Otsuka Y, Ito A, Matsumura S, Takeuchi M, Tanaka H. Effect of hydroxypropyl cellulose and hydroxypropyl methylcellulose on carbamazepine polymorphic transformation; attenuated total reflectance-infrared spectroscopy and chemoinformatics analysis. Colloid Polym Sci 2015;293:3471-78.

266.Ewing AV, Clarke GS, Kazarian SG. Stability of indomethacin with relevance to the release from amorphous solid dispersions studied with ATR-FTIR spectroscopic imaging. Eur J Pharm Sci 2014;60:64-71.

267.Lust A, Strachan CJ, Veski P, Aaltonen J, Heinämäki J, Yliruusi J, et al. Amorphous solid dispersions of piroxicam and Soluplus ${ }^{\circledR}$, Qualitative and quantitative analysis of piroxicam recrystallization during storage. Int $\mathbf{J}$ Pharm 2015;486:306-314.

268.Tian Y, Jones DS, Andrews GP. An Investigation into the role of polymeric carriers on crystal growth within amorphous solid dispersion systems. Mol Pharm 2015;12:1180-92.

269.Yang Z, Nollenberger K, Albers J, Moffat J, Craig D, Qi S. The effect of processing on the surface physical stability of amorphous solid dispersions. Eur $\mathbf{J}$ Pharm Biopharm 2014;88:897-908. 
270.Duarte Í, Santos JL, Pinto JF, Temtem M. Screening methodologies for the development of spray-dried amorphous solid dispersions. Pharm res 2015;32:222-37.

271.Yang JM, Zha L, Yu DG, Liu J. Coaxial electrospinning with acetic acid for preparing ferulic acid zein composite fibers with improved drug release profiles. Colloid Surf B 2012;102:737-43.

272.Rumondor ACF, Marsac PJ, Stanford LA, Taylor LS. Phase behavior of poly (vinylpyrrolidone) containing amorphous solid dispersions in the presence of moisture. Mol Pharm 2009;6:1492-1505.

273.Li XY, Wang X, Yu DG, Ye S, Kuang QK, Yi QW, et al. Electrospun borneolPVP nanocomposites. J Nanomater 2012;2012:DOI 10.1155/2012/731382.

274.Li L, li H, Qian Y, Li X, Singh GK, Zhong L, et al. Electrospun poly $(\varepsilon-$ caprolactone) silk fibroin core-sheath nanofibers and their potential applications in tissue engineering and drug release. Int J Biolog Macromol 2011;49:223-32.

275.Yu DG, Zhu LM, White K, Branford-White C. Electrospun nanofiber-based drug delivery systems. Health 2009;1:67-75.

276.Borbás E, Balogh A, Bocz K, Müller J, Kiserdei É, Vigh T, et al. In vitro dissolution-permeation evaluation of an electrospun cyclodextrin-based formulation of aripiprazole using $\mu$ FluxTM. Int J Pharm 2015;491:180-9.

277. Yang C, Yu DG, Pan D, Liu XK, Wang X, Bligh SWA, et al. Electrospun pHsensitive core-shell polymer nanocomposites fabricated using a tri-axial processes. Acta Biomater 2016;35:77-86.

278.Tran TH, Poudel BK, Marasini N, Chi SC, Choi HG, Yong CS, et al. Preparation and evaluation of raloxifene-loaded solid dispersion nanoparticle by spray-drying technique without an organic solvent. Int J Pharm 2013;443:50-7.

279.Wu L, Zhang J, Watanabe W. Physical and chemical stability of drug nanoparticles. Adv Drug Delivery Rev 2011;63:456-69.

280.Zussman E, Yarin A, Weihs D. A micro-aerodynamic decelerator based on permeable surfaces of nanofiber mats. Exp Fluid 2002;33:315-20.

281.Bevernage J, Forier T, Brouwers J, Tack J, Annaert P, Augustijns P. Excipient mediated supersaturation stabilization in human intestinal fluids. Mol pharm 2011;8: 564-70.

282. Borbás E, Sinkó B, Tsinman O, Tsinman K, Kiserdei E, Démuth B, et al. Investigation and mathematical description of the real driving force of passive transport of drug molecules from supersaturated solutions. Mol Pharm 2016;13: 3816-26.

283.Mishra DK, Dhote V, Bhargava A, Jain DK, Mishra PK. Amorphous solid dispersion technique for improved drug delivery, basics to clinical applications. Drug Deliv And Transl Res 2015;5:532-62.

284.Brough C, Williams RO. Amorphous solid dispersions and nano-crystal technologies for poorly water-soluble drug delivery. Int J Pharm 2013;453:15766.

285.Augustijns P, Brewster ME. Supersaturating drug delivery systems, Fast is not necessarily good enough. J Pharm Sci 2012;101:7-9.

286.Bevernage J, Brouwers J, Brewster ME, Augustijns P. Evaluation of gastrointestinal drug supersaturation and precipitation, Strategies and issues. Int $\mathbf{J}$ Pharm 2013;453:25-35.

287.Sun DD, Lee PI. Evolution of supersaturation of amorphous pharmaceuticals, The effect of rate of supersaturation generation. Mol. Pharm 2013;10:4330-6. 
288.Park K. Drug release mechanisms from amorphous solid dispersion. J Control Release 2015;211:171.

289.Sun DD, Lee PI. Probing the mechanisms of drug release from amorphous solid dispersions in medium-soluble and medium-insoluble carriers. J Control Release 2015;211:85-93.

290.Sun DD, Ju TCR, Lee PI. Enhanced kinetic solubility profiles of indomethacin amorphous solid dispersions in poly (2-hydroxyethyl methacrylate) hydrogels. Eur J Pharm Biopharm 2012;81:149-58.

291.Sun DD, Lee PI. Evolution of supersaturation of amorphous pharmaceuticals, nonlinear rate of supersaturation generation regulated by matrix diffusion. Mol Pharm 2015;12:1203-15.

292.Sun DD, Lee PI. Crosslinked hydrogels-a promising class of insoluble solidmolecular dispersion carriers for enhancing the delivery of poorly soluble drugs. Acta Pharm Sin B 2014;4:26-36.

293.Bandari S, Eaga CM, Thadishetty A, Yamsani MR. Formulation and evaluation of multiple tablets as a biphasic gastroretentive floating drug delivery system for fenoverine. Acta Pharm 2010;60:89-97.

294.Chen MC, Liang HF, Chiu YL, Chang Y, Wei HJ, Sung HW. A novel drug eluting stent spray-coated with multi-layers of collagen and sirolimus. J Control Release 2005;108:178-89.

295.Ofori-Kwakye K, Fell JT, Sharma HL, Smith AM. Gamma scintigraphic evaluation of film-coated tablets intended for colonic or biphasic release. Int $\mathbf{J}$ Pharm 2004;270:307-13.

296.Qian W, Yu DG, Li Y, Liao YZ, Wang X, Wang L. Dual drug release electrospun core-shell nanofibers with tunable dose in the second phase. Int J Mol Sci 2014; 15:774-86.

297.Huang LY, Branford-White C, Shen XX, Yu DG, Zhu LM. Time-Engineeringed Biphasic Drug Release by Electrospun Nanofiber Meshes. Int J Pharm 2012;436:88-96.

298.Song B, Wu C, Chang J. Dual. Drug Release from Electrospun Poly (lactic-coglycolic acid) Mesoporous Silica Nanoparticles Composite Mats with Distinct Release Profiles. Acta Biomater 2012;8:1901-7.

299.Yu DG, Li XY, Wang X, Yang JH, Bligh SWA, Williams GR. Nanofibers fabricated using triaxial electrospinning as zero order drug delivery systems. ACS Appl Mater Interfaces 2015;7:18891-7.

300.Song Y, Zemlyanov D, Chen X, Su Z, Nie H, Lubach JW, et al. Acid-base interactions in amorphous solid dispersions of lumefantrine prepared by spraydrying and hot-melt extrusion using X-ray photoelectron spectroscopy. Int $\mathbf{J}$ Pharm 2016;514: 456-64.

301.Stoddard RJ, Steger AL, Blakney AK, Woodrow KA. In pursuit of functional electrospun materials for clinical applications in humans. Therapeutic Del 2016; 7: 387-409.

302. Chen L, Okuda T, Lu XY, Chan HK. Amorphous powders for inhalation drug delivery. Adv Drug Delivery Rev 2016;100:102-15.

303.Qi S, Craig D. Recent developments in micro- and nanofabrication techniques for the preparation of amorphous pharmaceutical dosage forms. Adv Drug Delivery Rev 2016;100:67-84.

304.Yu DG, White K, Chatterton N, Li Y, Li LL, Wang X. Structural lipid nanoparticles self-assembled from electrospun core-shell polymeric nanocomposites. RSC Adv 2015;5:9462-6. 


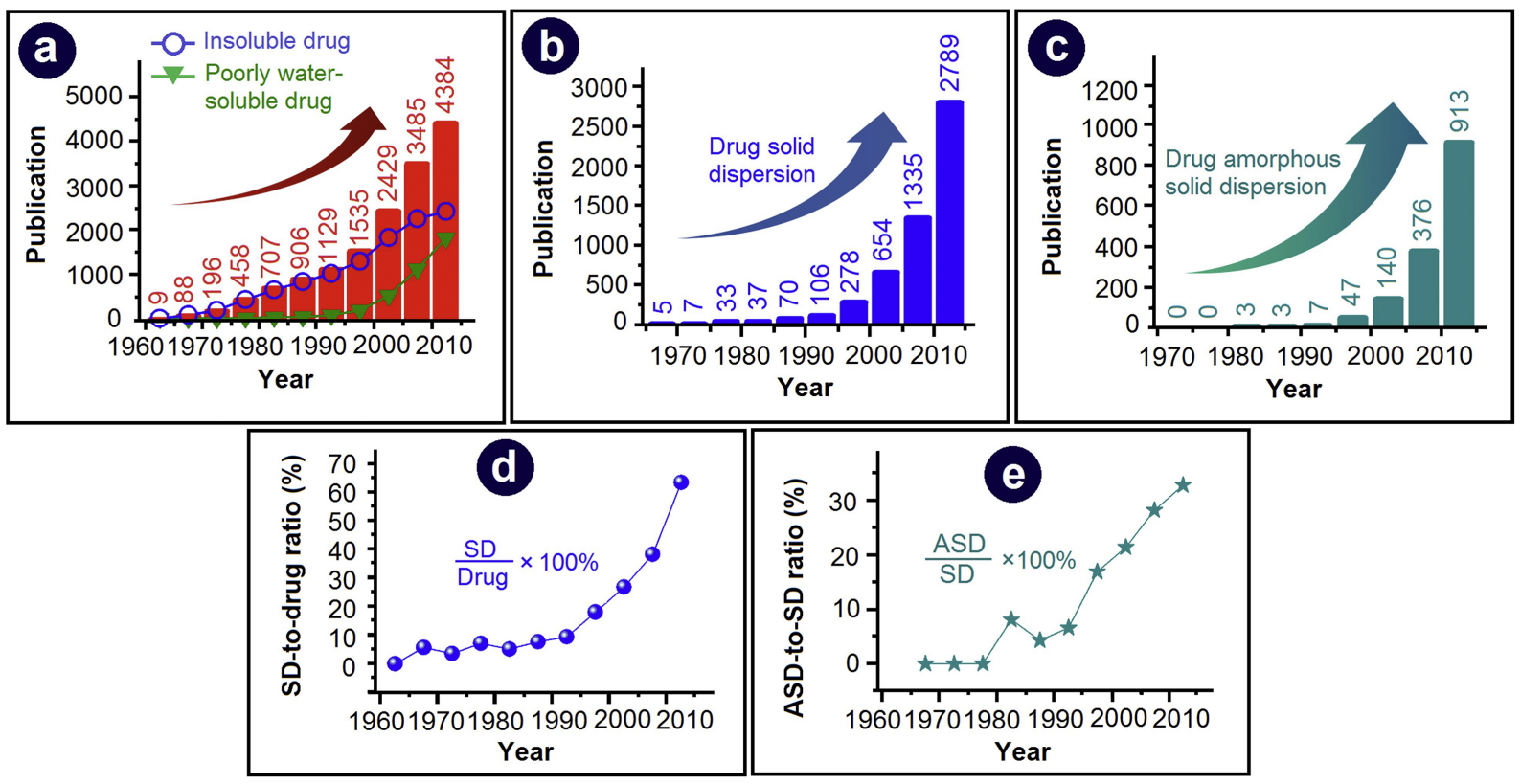

Figure 1 


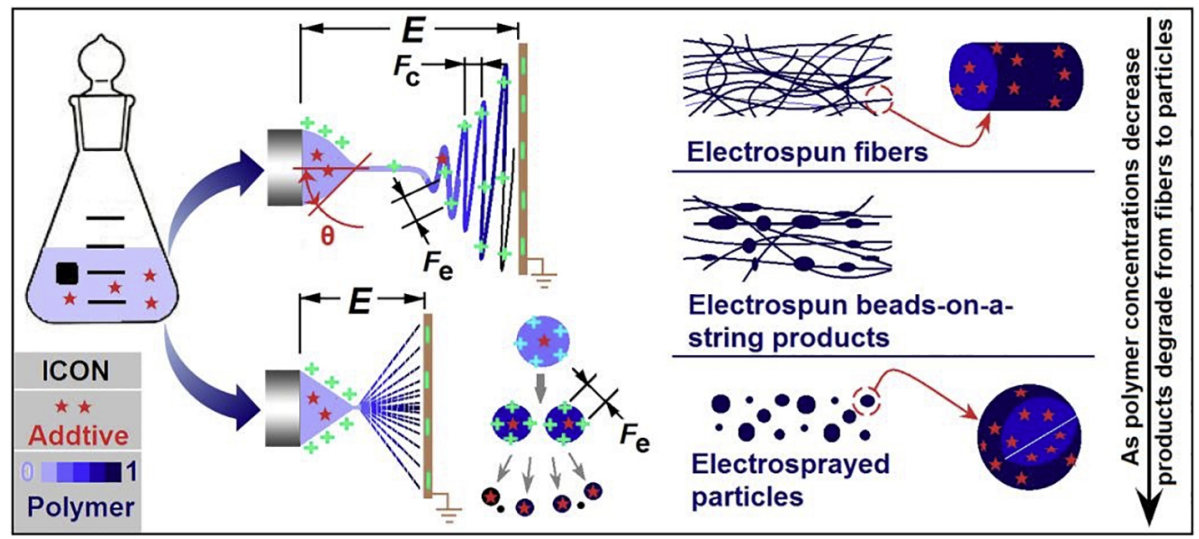

Figure 2 


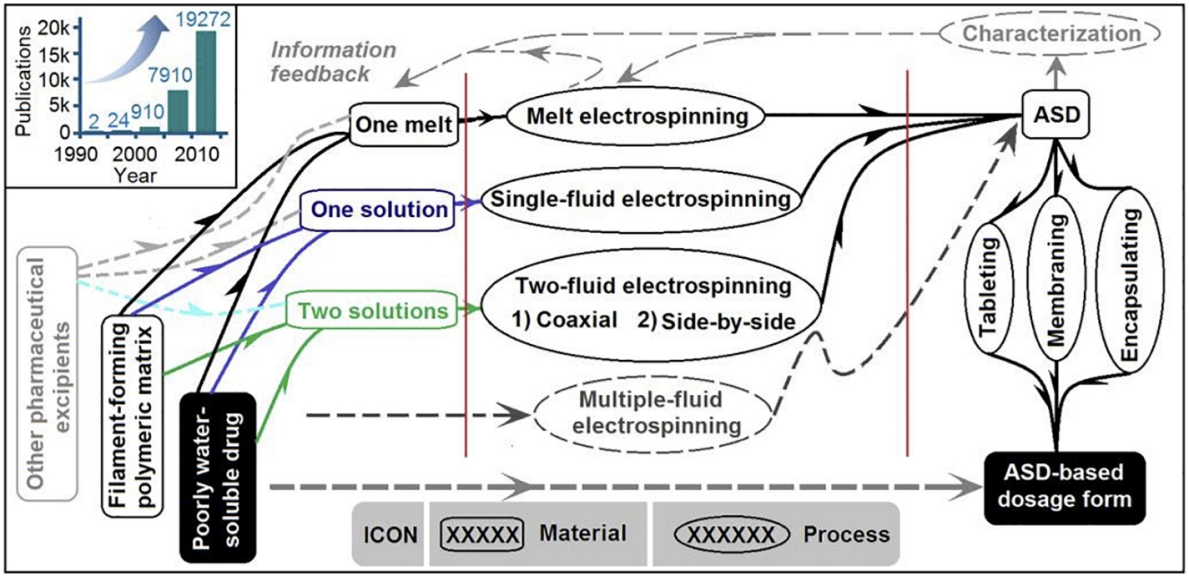

Figure 3 


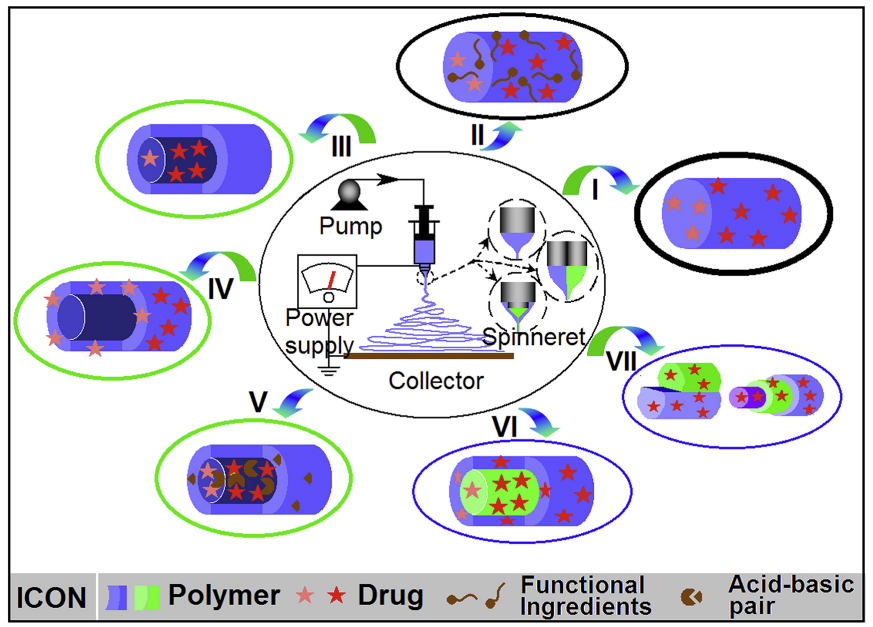

Figure 4 


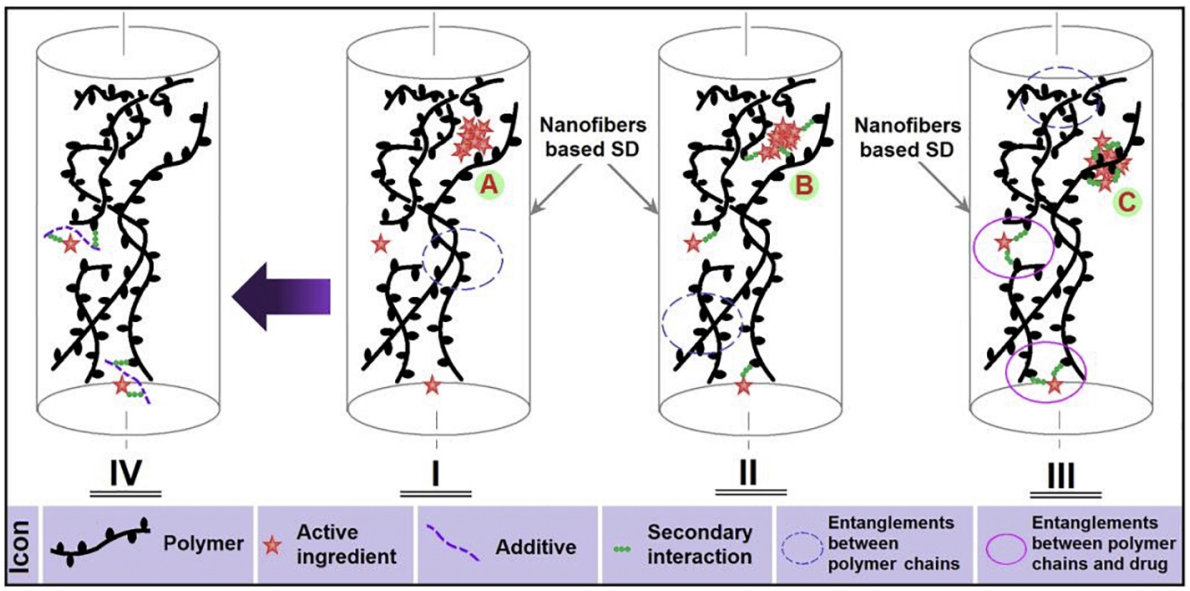

Figure 5 


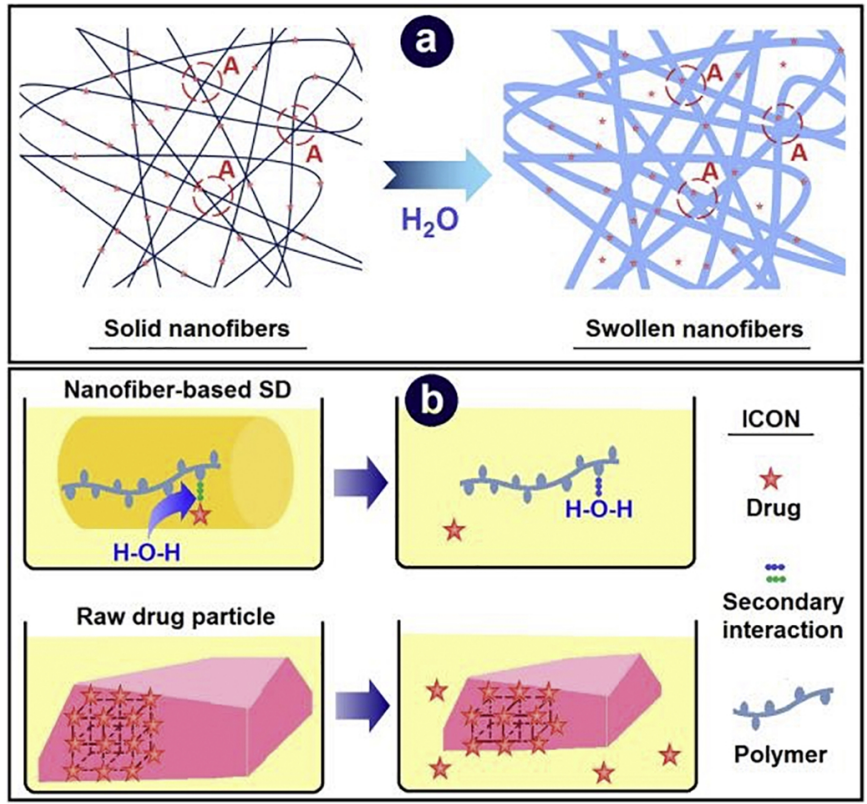

Figure 6 


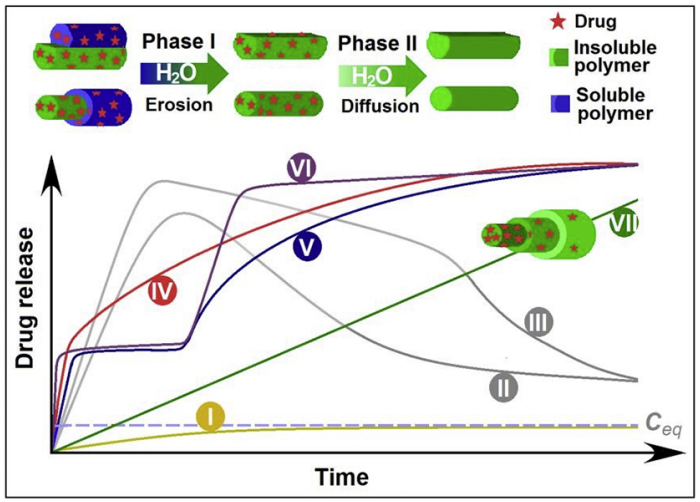

Figure 7 